\title{
Stability and convergence properties of forced infinite-dimensional discrete-time Lur'e systems
}

\author{
Max E. Gilmore* Chris Guiver*† Hartmut Logemann ${ }^{\ddagger}$
}

January 28, 2019

\begin{abstract}
Incremental stability and convergence properties for forced, infinite-dimensional, discretetime Lur'e systems are addressed. Lur'e systems have a linear and nonlinear component and arise as the feedback interconnection of a linear control system and a static nonlinearity. Discrete-time Lur'e systems arise in, for example, sampled-data control and integro-difference models. We provide conditions, reminiscent of classical absolute stability criteria, which are sufficient for a range of incremental stability properties and input-to-state stability (ISS). Consequences of our results include sufficient conditions for the converging-input convergingstate (CICS) property, and convergence to periodic solutions under periodic forcing.
\end{abstract}

Keywords: Absolute stability, converging-input converging-state property, integral projection models, incremental stability, infinite-dimensional discrete-time systems, input-to-state stability, Lur'e systems, sampled-data systems.

MSC(2010): 93C05, 93C10, 93C35, 93C55, 93C57, 93D05, 93D15, 93D20.

\section{Introduction}

In systems and control theory, feedback interconnections comprising a linear system in the forward path and a static nonlinearity in the feedback path, as shown in Figure 1.1, are commonly referred to as Lur'e systems. In this paper, we investigate certain stability and convergence properties of forced, infinite-dimensional, discrete-time Lur'e systems. Our focus is centred around incremental stability notions, input-to-state stability (ISS) and converging-input convergingstate (CICS) properties. The concept of ISS first appeared in 1989 in [45] and is a stability

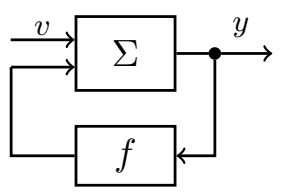

Figure 1.1: Lur'e system with linear part $\Sigma$, nonlinearity $f$, output $y$ and input $v$ concept pertaining to the states of (possibly nonlinear) control systems subject to external or

\footnotetext{
*Department of Mathematical Sciences, University of Bath, Claverton Down, Bath BA2 7AY, United Kingdom (m.e.gilmore@bath.ac.uk, c.guiver@bath.ac.uk)

${ }^{\dagger}$ Corresponding author

${ }^{\ddagger} 32$ Fairfield Avenue, Bath, BA1 6NH (h.logemann.varel@gmail.com)
} 
exogenous inputs. It ensures boundedness of the state in terms of the initial states and inputs, respectively, generalising the familiar additive estimate

$$
\|x(t)\| \leq \Gamma\left(\gamma^{t}\left\|x^{0}\right\|+\max _{0 \leq s \leq t-1}\|u(s)\|\right), \quad \forall t \in \mathbb{N},
$$

for some $\gamma \in(0,1)$ and $\Gamma>0$, for the state $x$ of the exponentially stable discrete-time linear system

$$
x(t+1)=A x(t)+u(t), \quad x(0)=x^{0}, \quad \forall t \in \mathbb{Z}_{+},
$$

with input $u$. Importantly, the constants $\gamma$ and $\Gamma$ are independent of $x^{0}$ and $u$. Since its inception, much attention has been devoted to ISS with numerous papers on the subject including, but not restricted to, $[10,19,20,21,22,23,24,46,48,49]$. The reader is referred to [8, 47] for overviews of key input-to-state stability ideas.

A related concept is incremental input-to-state stability ( $\delta$ ISS), which is simply an incremental version of the ISS concept and ensures boundedness of the difference of two state trajectories in terms of the difference of the initial conditions and the difference of the inputs. Evidently, for linear systems the notions of $\delta$ ISS and ISS coincide. The paper [1] constructs a suite of Lyapunov methods for $\delta$ ISS for finite-dimensional, continuous-time nonlinear control systems.

The study of the stability properties of Lur'e systems constitutes absolute stability theory which seeks to conclude stability of the feedback system shown in Figure 1.1 through the interplay of frequency domain properties of the linear system $\Sigma$ and the boundedness or sector properties of the nonlinearity $f$. Absolute stability theory is typically concerned with the development of criteria for global asymptotic stability of an equilibrium (typically zero) of unforced Lur'e systems [25, 27], or for $L^{2}$-stability in an input-output setting [50]. Classical absolute stability results include the circle criterion, the (complexified) Aizerman conjecture, and the Popov criterion [17, 25, 50].

As is well-known, global asymptotic or exponential stability of an equilibrium of an unforced nonlinear system does, in general, not guarantee any stability or boundedness properties of the system in the presence of forcing. A recent line of enquiry $[2,3,4,19,20,42,43,44]$ has been investigating to what extent classical absolute stability criteria can be modified to ensure ISS and state convergence properties of forced Lur'e systems. Indeed, a key finding has been that existing absolute stability criteria, under slightly stronger assumptions, are sufficient for ISS in many cases. The papers $[2,19,20,42,43,44]$ derive, in finite-dimensional settings, sufficient conditions for ISS which are reminiscent of the complexified Aizerman conjecture and the circle criterion. Furthermore, ISS properties underpin the paper [4], which considers the converginginput converging-state (CICS) property for finite-dimensional, continuous-time Lur'e systems.

Here we consider incremental ISS notions for forced infinite-dimensional discrete-time Lur'e systems. Our main result, Theorem 3.2, presents sufficient conditions for $\delta$ ISS in terms of a "nonlinear incremental ball condition" inspired by the complexified Aizerman conjecture. We appeal to exponential weighting and small-gain arguments to obtain a special type of $\delta$ ISS, that being, exponential $\delta$ ISS. We subsequently utilise $\delta$ ISS to obtain several different stability and convergence results. In particular, Corollary 3.3 provides an ISS result, Corollary 3.7 provides sufficient conditions for $\delta$ ISS which are reminiscent of the circle criterion, Theorem 4.3 gives sufficient conditions for the CICS property and Theorem 4.8 presents sufficient conditions for the existence of, and convergence to, periodic solutions under periodic forcing. At the time of writing, the study of ISS for infinite-dimensional, continuous-time control systems is an emerging research area, with papers including $[9,16,18,34,35,37,38]$, but to the best of our knowledge, there is no existing literature that overlaps with the current paper.

A motivation for the present study is its applicability in numerous areas. One such application is to infinite-dimensional sampled-data systems (see, for example, $[28,29,30,40]$ ). The sampled- 
data systems considered here are obtained from the feedback interconnection of a continuoustime infinite-dimensional system and a static nonlinearity using sample- and hold-operations. Theorem 6.1 provides conditions which guarantee that if the continuous-time feedback system is ISS, then the corresponding sampled-data system is ISS provided the sampling period is sufficiently small. A second class of examples arises in ecological modelling, and are so-called integral projection models (IPMs) [6, 12, 13], which are integro-difference models typically used for populations partitioned according to a continuous variable such as size or weight. The modelling assumption that there are both linear and nonlinear vital rates means that IPMs often naturally lead a Lur'e system structure. In Example 7.2, we demonstrate that, under natural assumptions, the theory developed in Sections 3 and 4 applies to a forced IPM for the plant Platte thistle, based on the model found in [39].

The paper is structured as follows. In Section 2 we gather relevant preliminary results regarding linear infinite-dimensional discrete-time systems. Section 3 contains the main results germane to ISS and $\delta$ ISS. Then, in Section 4 we utilise these results to yield convergence properties. Section 5 and Section 6 comprise applications of our earlier results in the form of 'four-block' systems and sampled-data systems, respectively. Finally, Section 7 contains detailed discussions of two examples.

Notation. Most notation we use is standard. The set of positive integers is denoted by $\mathbb{N}$, and $\mathbb{R}$ and $\mathbb{C}$ denote the fields of real and complex numbers, respectively. We set $\mathbb{R}_{+}:=\{r \in \mathbb{R}: r \geq 0\}$, $\mathbb{Z}_{+}=\mathbb{N} \cup\{0\}, \mathbb{C}_{0}=\{s \in \mathbb{C}: \operatorname{Re}(s)>0\}, \mathbb{D}_{\alpha}:=\{z \in \mathbb{C}:|z|<\alpha\}$ and $\mathbb{E}_{\alpha}:=\{z \in \mathbb{C}:|z|>\alpha\}$, where $\alpha>0$. For notational convenience, we let $\mathbb{D}:=\mathbb{D}_{1}$ and $\mathbb{E}:=\mathbb{E}_{1}$.

For normed spaces $V$ and $W$, we let $\mathcal{L}(V, W)$ denote the normed space of bounded linear operators from $V$ to $W$ and set $\mathcal{L}(V):=\mathcal{L}(V, V)$. Recall that an operator $A \in \mathcal{L}(V)$ is exponentially (or power) stable if the spectral radius of $A$ is strictly less than 1 . It is well known that $A \in \mathcal{L}(V)$ is exponentially stable if, and only if, there exist $M \geq 1$ and $\mu \in(0,1)$ such that

$$
\left\|A^{n}\right\| \leq M \mu^{n}, \quad \forall n \in \mathbb{Z}_{+} .
$$

In addition, the infimum of all $\mu>0$ satisfying (1.1) for some $M \geq 1$, is equal to the spectral radius of $A$. For $L \in \mathcal{L}(V, W)$ and $r>0$, we let

$$
\mathbb{B}(L, r):=\{M \in \mathcal{L}(V, W):\|M-L\|<r\},
$$

denote the open ball of radius $r$, centred at $L$. Throughout, for given normed spaces $V$ and $W$ we set

$$
\left\|\left(\begin{array}{l}
\eta \\
\xi
\end{array}\right)\right\|_{V \times W}:=\|\eta\|_{V}+\|\xi\|_{W}, \quad \forall\left(\begin{array}{l}
\eta \\
\xi
\end{array}\right) \in V \times W .
$$

For a Banach space $W$ and $\alpha>0$, we define the Hardy space

$$
H_{\alpha}^{\infty}(W):=\left\{g: \mathbb{E}_{\alpha} \rightarrow W \mid g \text { is holomorphic and bounded }\right\},
$$

with norm given by

$$
\|g\|_{H_{\alpha}^{\infty}}:=\sup _{z \in \mathbb{E}_{\alpha}}\|g(z)\|
$$

For ease of notation, we define

$$
H^{\infty}(W):=H_{1}^{\infty}(W) .
$$

For $t \in \mathbb{R}$, we define $\lfloor t\rfloor$ to be the greatest integer less than or equal to $t$ and $\lceil t\rceil$ to be the smallest integer greater than or equal to $t$, that is, the floor and ceiling of $t$, respectively. For a given $\tau \in \mathbb{Z}_{+}$, we define $\underline{\tau}:=\{0,1, \ldots, \tau\}, \bar{\tau}:=\{\tau, \tau+1, \ldots\}$ and the left-shift operator $\Lambda_{\tau}$ by $\left(\Lambda_{\tau} v\right)(t):=v(t+\tau)$ for every $t \in \mathbb{Z}_{+}$and every $v: \mathbb{Z}_{+} \rightarrow V$. For $v: \mathbb{Z}_{+} \rightarrow V$ and $t \in \mathbb{Z}_{+}$, we set

$$
\left(\pi_{t} v\right)(s):= \begin{cases}v(s), & \text { if } s \in \underline{t} \\ 0, & \text { otherwise. }\end{cases}
$$


For $p \in[1, \infty)$, let $\ell^{p}(V)$ be the space of functions $x: \mathbb{Z}_{+} \rightarrow V$ such that $\left(\sum_{k=0}^{\infty}\|x(t)\|_{V}^{p}\right)^{1 / p}<\infty$, with norm $\|x\|_{\ell^{p}}:=\left(\sum_{k=0}^{\infty}\|x(t)\|_{V}^{p}\right)^{1 / p}$, and let $\ell^{\infty}(V)$ be the space of functions $x: \mathbb{Z}_{+} \rightarrow V$ such that $\sup _{t \in \mathbb{Z}_{+}}\|x(t)\|_{V}<\infty$, with norm $\|x\|_{\ell^{\infty}}:=\sup _{t \in \mathbb{Z}_{+}}\|x(t)\|_{V}$. Furthermore, for $\rho>0$, we define the weighted $\ell^{2}$ space

$$
\ell_{\rho}^{2}(V):=\left\{x \in \ell^{2}(V):\left(\sum_{t=0}^{\infty}\|x(t)\|^{2} \rho^{2 t}\right)^{1 / 2}<\infty\right\},
$$

with norm $\|\cdot\|_{\ell_{\rho}^{2}}$, defined by

$$
\|x\|_{\ell_{\rho}^{2}}:=\left(\sum_{t=0}^{\infty}\|x(t)\|^{2} \rho^{2 t}\right)^{1 / 2}, \quad x \in \ell_{\rho}^{2}(V) .
$$

We denote the set of functions from $\mathbb{Z}_{+} \rightarrow V$ by $V^{\mathbb{Z}_{+}}$and the set of continuous functions $\mathbb{R}_{+} \rightarrow V$ by $C\left(\mathbb{R}_{+}, V\right)$. Finally, for $v^{\mathrm{e}} \in V$, we will abuse notation and interchangeably write $v^{\mathrm{e}}$ to denote an element of $V$ and the constant function $\mathbb{Z}_{+} \rightarrow V$ with value $v^{\mathrm{e}}$.

\section{Preliminaries}

To begin with, we present some preliminary results regarding the following linear difference equation

$$
\left.\begin{array}{rl}
x^{+} & =A x+B u+B_{\mathrm{e}} v \\
y & =C x+D u+D_{\mathrm{e}} v
\end{array}\right\}
$$

where

$$
\left(A, B, B_{\mathrm{e}}, C, D, D_{\mathrm{e}}\right) \in \mathcal{L}(X) \times \mathcal{L}(U, X) \times \mathcal{L}(V, X) \times \mathcal{L}(X, Y) \times \mathcal{L}(U, Y) \times \mathcal{L}(V, Y)=: \mathbb{L},
$$

$u \in U^{\mathbb{Z}_{+}}$and $v \in V^{\mathbb{Z}_{+}}$. Here $X$ and $V$ are complex Banach spaces and $U$ and $Y$ are complex Hilbert spaces. The variables $x$ and $y$ in (2.1) are called the state and output, respectively, and $u$ and $v$ are inputs. Occasionally, it will be convenient to identify the linear system (2.1) and the sextuple $\left(A, B, B_{\mathrm{e}}, C, D, D_{\mathrm{e}}\right)$ and to refer to the linear system $\left(A, B, B_{\mathrm{e}}, C, D, D_{\mathrm{e}}\right)$. For ease of notation, we set $\Sigma:=\left(A, B, B_{\mathrm{e}}, C, D, D_{\mathrm{e}}\right) \in \mathbb{L}$.

Before continuing, it is worth noting that (2.1) encompasses other seemingly more general linear systems. For instance, the linear system

$$
\begin{aligned}
x^{+} & =A x+B u+v^{1}, \\
y & =C x+D u+v^{2},
\end{aligned}
$$

where $v^{1} \in X^{\mathbb{Z}_{+}}$and $v^{2} \in Y^{\mathbb{Z}_{+}}$, is a special case of (2.1) with $V=X \times Y, B_{\mathrm{e}}=\left(\begin{array}{ll}I & 0\end{array}\right)$, $D_{\mathrm{e}}=\left(\begin{array}{ll}0 & I\end{array}\right)$ and $v=\left(\begin{array}{c}v^{1} \\ v^{2}\end{array}\right)$.

We record some definitions associated with (2.1). First, we define the behaviour of (2.1) as

$$
\mathcal{B}^{\text {lin }}:=\left\{(u, v, x, y) \in U^{\mathbb{Z}_{+}} \times V^{\mathbb{Z}_{+}} \times X^{\mathbb{Z}_{+}} \times Y^{\mathbb{Z}_{+}}:(u, v, x, y) \text { satisfies }(2.1)\right\},
$$

and set $\mathbf{G}(z)=C(z I-A)^{-1} B+D$, a $\mathcal{L}(U, Y)$-valued function of the complex variable $z$, the so-called transfer function of (2.1) from $u$ to $y$. If $\mu$ denotes the exponential growth constant of $A$, then $\mathbf{G} \in H_{\alpha}^{\infty}(\mathcal{L}(U, Y))$ for all $\alpha>\mu$, meaning that $\mathbf{G}$ is bounded and holomorphic on the exterior of any open disc in $\mathbb{C}$ centred at 0 with radius greater than $\mu$. We say that $\Sigma \in \mathbb{L}$ is 
stabilisable (respectively detectable) if $(A, B, C, D)$ is (exponentially) stabilisable (respectively detectable).

We define the set of admissible feedback operators by

$$
\mathbb{A}(D):=\{L \in \mathcal{L}(Y, U): I-D L \text { is invertible }\} .
$$

For $L \in \mathbb{A}(D)$, we set

$$
\left.\begin{array}{rl}
A^{L}:=A+B L(I-D L)^{-1} C, & B^{L}:=B(I-L D)^{-1}, \\
C^{L}:=(I-D L)^{-1} C, & D^{L}:=(I-D L)^{-1} D .
\end{array}\right\}
$$

We denote by $\mathbf{G}^{L}$ the transfer function of $\left(A^{L}, B^{L}, C^{L}, D^{L}\right)$, that is,

$$
\mathbf{G}^{L}(z)=C^{L}\left(z I-A^{L}\right)^{-1} B^{L}+D^{L}=\mathbf{G}(z)(I-L \mathbf{G}(z))^{-1},
$$

where the second equality follows easily from (2.2). For given $L \in \mathbb{A}(D)$, the operators $A^{L}, B^{L}, C^{L}$ and $D^{L}$ arise by applying the feedback $u=L y+\tilde{u}$ to $(2.1)$ where $\tilde{u} \in U^{\mathbb{Z}_{+}}$. The transfer function from $\tilde{u}$ to $y$ is $\mathbf{G}^{L}$. Finally, we define the set of (complex) stabilising feedback operators

$$
\mathbb{S}(\mathbf{G}):=\left\{M \in \mathbb{A}(D): \mathbf{G}^{M} \in H^{\infty}(\mathcal{L}(U, Y))\right\} .
$$

We next state three lemmas which underpin our development. The first lemma is a discrete-time version of [15, Proposition 5.6]. We omit the proof, since it is similar to that in [15].

Lemma 2.1. Let $\Sigma \in \mathbb{L}, L \in \mathbb{A}(D)$ and $r>0$. We have $\mathbb{B}(L, r) \subseteq \mathbb{S}(\mathbf{G})$ if, and only if, $\left\|\mathbf{G}^{L}\right\|_{H^{\infty}} \leq 1 / r$.

The following lemma relates $\mathcal{B}^{\text {lin }}$ to the behaviour of a certain feedback system, a process often called 'loop shifting' in control theory. The proof is relegated to Appendix A.

Lemma 2.2. Let $\Sigma \in \mathbb{L}$ and $L \in \mathbb{A}(D)$. The quadruple $(u, v, x, y)$ is in $\mathcal{B}^{\text {lin }}$ if, and only if, $(u, v, x, y)$ satisfies

$$
\begin{aligned}
x^{+} & =A^{L} x+B^{L}(u-L y)+\left(B_{\mathrm{e}}+B^{L} L D_{\mathrm{e}}\right) v, \\
y & =C^{L} x+D^{L}(u-L y)+(I-D L)^{-1} D_{\mathrm{e}} v .
\end{aligned}
$$

We set $\Sigma^{L}:=\left(A^{L}, B^{L}, B_{\mathrm{e}}+B^{L} L D_{\mathrm{e}}, C^{L}, D^{L},(I-D L)^{-1} D_{\mathrm{e}}\right) \in \mathbb{L}$.

We now state the third lemma, the proof of which is elementary, and is therefore omitted.

Lemma 2.3. Let $\Sigma \in \mathbb{L}$ and assume that $A$ is exponentially stable. Then there exist $c_{1}, c_{2}, c_{3}>0$ such that, for every $(u, v, x, y) \in \mathcal{B}^{\text {lin }}$, we have

$$
\text { and } \begin{aligned}
& \left\|\pi_{t} x\right\|_{\ell^{2}} \leq c_{1}\|x(0)\|_{X}+c_{2}\left\|\pi_{t-1} u\right\|_{\ell^{2}}+c_{3}\left\|\pi_{t-1} v\right\|_{\ell^{2}}, \quad \forall t \in \mathbb{N}, \\
& \quad\left\|\pi_{t} y\right\|_{\ell^{2}} \leq c_{1}\|x(0)\|_{X}+\|\mathbf{G}\|_{H^{\infty}}\left\|\pi_{t} u\right\|_{\ell^{2}}+c_{3}\left\|\pi_{t} v\right\|_{\ell^{2}}, \quad \forall t \in \mathbb{Z}_{+} .
\end{aligned}
$$

The nonlinear control systems considered in the current paper are given by the interconnection of (2.1) with the nonlinear feedback $u=f(y+w)$ for some $f: Y \rightarrow U$, where $w \in Y^{\mathbb{Z}_{+}}$is an output disturbance (see Figure 2.1).

Namely, we study

$$
\left.\begin{array}{rl}
x^{+} & =A x+B u+B_{\mathrm{e}} v, \\
y & =C x+D u+D_{\mathrm{e}} v, \\
u & =f(y+w),
\end{array}\right\}
$$




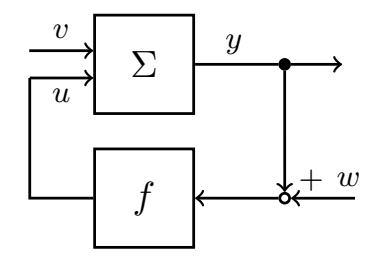

Figure 2.1: Block diagram of the feedback interconnection of (2.1) with $u=f(y+w)$.

where $\Sigma \in \mathbb{L}, v \in V^{\mathbb{Z}_{+}}, w \in Y^{\mathbb{Z}_{+}}$and $f: Y \rightarrow U$.

We note that in the case that $I-D f$ is invertible, then (2.3) may be expressed more succinctly as

$$
x^{+}=A x+B f\left((I-D f)^{-1}\left(C x+D_{\mathrm{e}} v+w\right)\right)+B_{\mathrm{e}} v .
$$

We define the behaviour of $(2.3)$ as

$$
\mathcal{B}:=\left\{(v, w, x, y) \in V^{\mathbb{Z}_{+}} \times Y^{\mathbb{Z}_{+}} \times X^{\mathbb{Z}_{+}} \times Y^{\mathbb{Z}_{+}}:(v, w, x, y) \text { satisfies }(2.3)\right\} .
$$

Note that if $f(0)=0$, then $(0,0,0,0) \in \mathcal{B}$. An important observation is that $\mathcal{B}$ is left-shift invariant:

$$
(v, w, x, y) \in \mathcal{B} \quad \Rightarrow \quad\left(\Lambda_{\tau} v, \Lambda_{\tau} w, \Lambda_{\tau} x, \Lambda_{\tau} y\right) \in \mathcal{B}, \quad \forall \tau \in \mathbb{Z}_{+} .
$$

Associated with (2.3) is the following initial-value-problem.

$$
\left.\begin{array}{rl}
x^{+} & =A x+B u+B_{\mathrm{e}} v, \quad x(0)=x^{0} \in X, \\
y & =C x+D u+D_{\mathrm{e}} v, \\
u & =f(y+w) .
\end{array}\right\}
$$

For a given $x^{0} \in X, v \in V^{\mathbb{Z}_{+}}$and $w \in Y^{\mathbb{Z}_{+}}$, we say that $(x, y) \in X^{\mathbb{Z}_{+}} \times Y^{\mathbb{Z}_{+}}$is a solution of $(2.5)$ if $x(0)=x^{0}$ and $(v, w, x, y) \in \mathcal{B}$. It is straightforward to prove that if the map $I-D f$ is surjective, then, for a given $x^{0} \in X, v \in V^{\mathbb{Z}_{+}}$and $w \in Y^{\mathbb{Z}_{+}}$, solutions to (2.5) exist. It is also straightforward to prove that if $I-D f$ is injective, then, for a given $x^{0} \in X, v \in V^{\mathbb{Z}_{+}}$ and $w \in Y^{\mathbb{Z}_{+}}$, there is at most one solution of (2.5). We note that both of these properties are evidently satisfied if $D=0$. The following example demonstrates that each of the previous conclusions need not hold if injectivity or surjectivity of $I-D f$ are respectively dropped.

Example 2.4. Consider (2.5) in the finite-dimensional single-input single-output case wherein $X=U=V=Y=\mathbb{C}$ and $A=B=C=D=B_{\mathrm{e}}=D_{\mathrm{e}}=1$. Thus (2.5) becomes

$$
\left.\begin{array}{rl}
x^{+} & =x+f(y+w)+v, \quad x(0)=x^{0} \in X, \\
y & =x+f(y+w)+v .
\end{array}\right\}
$$

i) Let $f(z)=z-e^{z}$, for all $z \in \mathbb{C}$, and let $x^{0}=0$ and $v(0)=w(0)=0$. Suppose that $(x, y)$ is a solution to $(2.6)$. Then, in particular, $y(0)=f(y(0))$ and so, $e^{y(0)}=0$, which is impossible. Therefore, for $x^{0}=0$ and $v(0)=w(0)=0,(2.6)$ has no solutions.

ii) Let $f(s)=2 s-s^{2}-s^{3}$, for all $s \in \mathbb{C}$. Note that it is easy to verify that there exist multiple solutions to (2.6) with $x^{0}=1$ and $v=w=0$.

\section{Exponential incremental stability}

In this section, we recall notions of exponential input-to-state stability, define notions of exponential incremental input-to-state stability and present a condition which guarantees that the 
Lur'e system (2.3) is exponentially incrementally input-to-state/output stable. Throughout the following definitions we let $\Sigma \in \mathbb{L}$ and $f: Y \rightarrow U$. A quadruple $\left(v^{\mathrm{e}}, w^{\mathrm{e}}, x^{\mathrm{e}}, y^{\mathrm{e}}\right) \in V \times Y \times X \times Y$ is called an equilibrium quadruple of $(2.3)$ if $\left(v^{\mathrm{e}}, w^{\mathrm{e}}, x^{\mathrm{e}}, y^{\mathrm{e}}\right) \in \mathcal{B}$. An equilibrium quadruple $\left(v^{\mathrm{e}}, w^{\mathrm{e}}, x^{\mathrm{e}}, y^{\mathrm{e}}\right)$ is said to be exponentially input-to-state stable (ISS) if there exist $c>0$ and $a \in(0,1)$ such that, for all $(v, w, x, y) \in \mathcal{B}$ we have

$$
\left\|x(t)-x^{\mathrm{e}}\right\|_{X} \leq c\left(a^{t}\left\|x(0)-x^{\mathrm{e}}\right\|_{X}+\max _{s \in \underline{t-1}}\left\|\left(\begin{array}{c}
v(s) \\
w(s)
\end{array}\right)-\left(\begin{array}{c}
v^{\mathrm{e}} \\
w^{\mathrm{e}}
\end{array}\right)\right\|_{V \times Y}\right), \quad \forall t \in \mathbb{N} .
$$

Further, an equilibrium quadruple is said to be exponentially input-to-state/output stable (ISOS) if there exist $c>0$ and $a \in(0,1)$ such that, for all $(v, w, x, y) \in \mathcal{B},(3.1)$ holds and

$$
\left\|y(t)-y^{\mathrm{e}}\right\|_{Y} \leq c\left(a^{t}\left\|x(0)-x^{\mathrm{e}}\right\|_{X}+\max _{s \in \underline{t}}\left\|\left(\begin{array}{c}
v(s) \\
w(s)
\end{array}\right)-\left(\begin{array}{c}
v^{\mathrm{e}} \\
w^{\mathrm{e}}
\end{array}\right)\right\|_{V \times Y}\right), \quad \forall t \in \mathbb{Z}_{+} .
$$

We say that (2.3) is exponentially ISS (respectively, ISOS) if $(0,0,0,0)$ is an exponentially ISS (respectively, ISOS) equilibrium quadruple of (2.3).

The following example demonstrates a situation where (2.3) is exponentially ISS but not exponentially ISOS.

Example 3.1. Consider (2.3) where $X=U=V=Y=\mathbb{C}^{2}$, $f$ is the identity map, $A \in \mathbb{R}^{2 \times 2}$ is exponentially stable,

$$
B:=\left(\begin{array}{ll}
0 & 1 \\
0 & 0
\end{array}\right), \quad C:=\left(\begin{array}{ll}
1 & 0 \\
0 & 0
\end{array}\right)=: D, \quad \text { and } \quad B_{\mathrm{e}}:=\left(\begin{array}{ll}
1 & 0 \\
0 & 1
\end{array}\right)=: D_{\mathrm{e}} .
$$

Since $B C=0=B D$ and $A$ is exponentially stable, it is easily checked that (2.3) is exponentially ISS. We shall now show that (2.3) is not exponentially ISOS. To this end, first let $x^{0} \in \mathbb{R}^{2}$ and let $x:=\left(x^{1}, x^{2}\right)^{T} \in\left(\mathbb{R}^{2}\right)^{\mathbb{Z}_{+}}$be such that

$$
x^{+}=A x, \quad x(0)=x^{0} .
$$

By setting $w:=\left(-x^{1}, 0\right)^{T} \in\left(\mathbb{R}^{2}\right)^{\mathbb{Z}_{+}}$and $y:=\left(y^{1}, 0\right)^{T} \in\left(\mathbb{R}^{2}\right)^{\mathbb{Z}_{+}}$for arbitrary $y^{1} \in \mathbb{R}^{\mathbb{Z}_{+}}$, we see that

$$
C x+D(y+w)=\left(\begin{array}{c}
x^{1} \\
0
\end{array}\right)+\left(\begin{array}{c}
y^{1}-x^{1} \\
0
\end{array}\right)=\left(\begin{array}{c}
y^{1} \\
0
\end{array}\right)=y
$$

and, since $B C=0=B D$ and $B w=0$,

$$
x^{+}=A x=A x+B(C x+D(y+w)+w)=A x+B(y+w) .
$$

Hence, $(0, w, x, y) \in \mathcal{B}$. This holds for any $y^{1} \in \mathbb{R}^{\mathbb{Z}_{+}}$and so $(2.3)$ is not ISOS.

For a non-empty subset $S \subseteq Y$, we define the following sub-behaviour of (2.3)

$$
\mathcal{B}^{S}:=\left\{(v, w, x, y) \in \mathcal{B}: y(t)+w(t) \in S, \forall t \in \mathbb{Z}_{+}\right\},
$$

and note that $\mathcal{B}^{Y}=\mathcal{B}$.

We say that (2.3) is exponentially incrementally input-to-state stable ( $\delta I S S)$ with respect to the non-empty sets $S_{1}, S_{2} \subseteq Y$ if there exist $c>0$ and $a \in(0,1)$ such that, for all $\left(v_{1}, w_{1}, x_{1}, y_{1}\right) \in$ $\mathcal{B}^{S_{1}}$ and for all $\left(v_{2}, w_{2}, x_{2}, y_{2}\right) \in \mathcal{B}^{S_{2}}$ we have

$$
\left\|x_{1}(t)-x_{2}(t)\right\|_{X} \leq c\left(a^{t}\left\|x_{1}(0)-x_{2}(0)\right\|_{X}+\max _{s \in \underline{t-1}}\left\|\left(\begin{array}{c}
v_{1}(s) \\
w_{1}(s)
\end{array}\right)-\left(\begin{array}{c}
v_{2}(s) \\
w_{2}(s)
\end{array}\right)\right\|_{V \times Y}\right), \quad \forall t \in \mathbb{N} .
$$


Further, (2.3) is exponentially incrementally input-to-state/output stable (8ISOS) with respect to the sets $S_{1}$ and $S_{2}$, if there exist $c>0$ and $a \in(0,1)$ such that, for all $\left(v_{1}, w_{1}, x_{1}, y_{1}\right) \in \mathcal{B}^{S_{1}}$ and for all $\left(v_{2}, w_{2}, x_{2}, y_{2}\right) \in \mathcal{B}^{S_{2}},(3.2)$ holds and

$$
\left\|y_{1}(t)-y_{2}(t)\right\|_{Y} \leq c\left(a^{t}\left\|x_{1}(0)-x_{2}(0)\right\|_{X}+\max _{s \in \underline{t}}\left\|\left(\begin{array}{c}
v_{1}(s) \\
w_{1}(s)
\end{array}\right)-\left(\begin{array}{c}
v_{2}(s) \\
w_{2}(s)
\end{array}\right)\right\|_{V \times Y}\right), \quad \forall t \in \mathbb{Z}_{+} .
$$

In the case that $f(0)=0$, if (2.3) is $\delta$ ISS (respectively, $\delta$ ISOS) with respect to $S_{1}:=Y$ and $S_{2}:=\{0\}$, then, trivially, (2.3) is also ISS (respectively, ISOS). The Lur'e system (2.3) is said to be exponentially $\delta$ ISS or exponentially $\delta$ ISOS if $S_{1}=S_{2}=Y$ in the above respective definitions. Trivially, exponential $\delta$ ISOS with respect to $S_{1}$ and $S_{2}$ implies exponential $\delta$ ISS with respect to the same sets. The following theorem is the main result of this section.

Theorem 3.2. Let $\Sigma \in \mathbb{L}$ be stabilisable and detectable and let $S_{1}, S_{2} \subseteq Y$ be non-empty. Assume that $r>0$ and $K \in \mathcal{L}(Y, U)$ satisfy $\mathbb{B}(K, r) \subseteq \mathbb{S}(\mathbf{G})$ and that there exists $\delta \in(0, r)$ such that

$$
\|f(\xi)-f(\zeta)-K(\xi-\zeta)\|_{U} \leq(r-\delta)\|\xi-\zeta\|_{Y}, \quad \forall \xi \in S_{1}, \forall \zeta \in S_{2} .
$$

Then the following hold.

(i) There exist constants $a>0, b>0$ and $\omega>1$ such that, for all $\left(v_{1}, w_{1}, x_{1}, y_{1}\right) \in \mathcal{B}^{S_{1}}$, $\left(v_{2}, w_{2}, x_{2}, y_{2}\right) \in \mathcal{B}^{S_{2}}$, and all $\rho \in[1, \omega]$, we have

$$
\left\|\pi_{t}\left(x_{1}-x_{2}\right)\right\|_{\ell_{\rho}^{2}} \leq a\left(\left\|x_{1}(0)-x_{2}(0)\right\|_{X}+\left\|\pi_{t-1}\left(\begin{array}{c}
v_{1}-v_{2} \\
w_{1}-w_{2}
\end{array}\right)\right\|_{\ell_{\rho}^{2}}\right), \quad \forall t \in \mathbb{N},
$$

and

$$
\left\|\pi_{t}\left(y_{1}-y_{2}\right)\right\|_{\ell_{\rho}^{2}} \leq b\left(\left\|x_{1}(0)-x_{2}(0)\right\|_{X}+\left\|\pi_{t}\left(\begin{array}{c}
v_{1}-v_{2} \\
w_{1}-w_{2}
\end{array}\right)\right\|_{\ell_{\rho}^{2}}\right), \quad \forall t \in \mathbb{Z}_{+} .
$$

(ii) For $q \in[2, \infty]$, there exist constants $c>0, d>0$ and $\theta \in(0,1)$ such that, for all $\left(v_{1}, w_{1}, x_{1}, y_{1}\right) \in \mathcal{B}^{S_{1}}$ and $\left(v_{2}, w_{2}, x_{2}, y_{2}\right) \in \mathcal{B}^{S_{2}}$, we have

$$
\left\|x_{1}(t)-x_{2}(t)\right\|_{X} \leq c\left(\theta^{t}\left\|x_{1}(0)-x_{2}(0)\right\|_{X}+\left\|\pi_{t-1}\left(\begin{array}{c}
v_{1}-v_{2} \\
w_{1}-w_{2}
\end{array}\right)\right\|_{\ell^{q}}\right), \quad \forall t \in \mathbb{N},
$$

and

$$
\left\|y_{1}(t)-y_{2}(t)\right\|_{Y} \leq d\left(\theta^{t}\left\|x_{1}(0)-x_{2}(0)\right\|_{X}+\left\|\pi_{t}\left(\begin{array}{c}
v_{1}-v_{2} \\
w_{1}-w_{2}
\end{array}\right)\right\|_{\ell^{q}}\right), \quad \forall t \in \mathbb{Z}_{+} .
$$

Here $c$ and $d$ depend on $q$, but $\theta$ does not.

As an immediate consequence of Theorem 3.2, by taking $q=\infty$, we obtain the following corollary.

Corollary 3.3. Under the assumptions of Theorem 3.2, the Lur'e system (2.3) is exponentially $\delta I S O S$ with respect to $S_{1}$ and $S_{2}$. In particular, the following statements hold.

(i) If (3.3) holds with $S_{1}=S_{2}=Y$, then (2.3) is exponentially IISOS.

(ii) If $\left(v^{\mathrm{e}}, w^{\mathrm{e}}, x^{\mathrm{e}}, y^{\mathrm{e}}\right)$ is an equilibrium quadruple of (2.3) and (3.3) holds with $S_{1}=Y$ and $S_{2}=$ $\left\{y^{\mathrm{e}}+w^{\mathrm{e}}\right\}$, then $\left(v^{\mathrm{e}}, w^{\mathrm{e}}, x^{\mathrm{e}}, y^{\mathrm{e}}\right)$ is an exponentially ISOS equilibrium quadruple of (2.3). 
Proof of Theorem 3.2. The proof uses a combination of small-gain and exponential weighting arguments. Since $\Sigma$ is stabilisable and detectable, it follows that $\Sigma^{K}$ is as well. Moreover, since $\mathbf{G}^{K} \in H^{\infty}(\mathcal{L}(U, Y))$, it follows that $A^{K}$ is exponentially stable, with exponential growth constant $\mu \in(0,1)$. Let $\alpha \in(\mu, 1)$ so that $\mathbf{G}^{K} \in H_{\alpha}^{\infty}(\mathcal{L}(U, Y))$ and consider $\mathbf{G}^{K}$ on the closed annulus $\mathcal{A}:=\{z \in \mathbb{C}: \beta \leq|z| \leq 1\}$, where $\beta \in(\alpha, 1)$. Owing to the continuity of $\mathbf{G}^{K}$ on $\mathbb{E}_{\alpha}$, $\mathbf{G}^{K}$ is uniformly continuous on $\mathcal{A}$. Thus, there exists $\gamma \in(0,1-\beta)$ such that for all $s_{1}, s_{2} \in \mathcal{A}$ with $\left|s_{1}-s_{2}\right|<\gamma$, we have

$$
\left\|\mathbf{G}^{K}\left(s_{1}\right)-\mathbf{G}^{K}\left(s_{2}\right)\right\|<\frac{1}{r-\delta / 2}-\frac{1}{r} .
$$

Invoking Lemma 2.1, we have that $\left\|\mathbf{G}^{K}\right\|_{H^{\infty}} \leq 1 / r$. Thus, for all $s \in \mathbb{C}$ with $1-\gamma<|s| \leq 1$,

$$
\begin{aligned}
\left\|\mathbf{G}^{K}(s)\right\| & \leq\left\|\mathbf{G}^{K}(s)-\mathbf{G}^{K}\left(s^{*}\right)\right\|+\left\|\mathbf{G}^{K}\left(s^{*}\right)\right\| \\
& <\frac{1}{r-\delta / 2}-\frac{1}{r}+\frac{1}{r}=\frac{1}{r-\delta / 2},
\end{aligned}
$$

where $s^{*}=s /|s|$. Hence, for $\gamma^{*} \in(1-\gamma, 1)$

$$
\sup _{s \in \mathbb{E}_{\gamma^{*}}}\left\|\mathbf{G}^{K}(s)\right\| \leq \frac{1}{r-\delta / 2}<\frac{1}{r-\delta} .
$$

To prove statement (i), set $\omega:=1 / \gamma^{*}>1$ and let $\rho \in[1, \omega]$. Define $\mathbf{H}(s):=\mathbf{G}^{K}(s / \rho)$ to obtain

$$
\|\mathbf{H}\|_{H^{\infty}}=\sup _{s \in \mathbb{E}}\|\mathbf{H}(s)\| \leq \sup _{s \in \mathbb{E}_{\gamma^{*}}}\left\|\mathbf{G}^{K}(s)\right\|<\frac{1}{r-\delta} .
$$

By the choice of $\rho$, we have that

$$
\rho \mu<\frac{\mu}{1-\gamma}<\frac{\mu}{\beta}<1
$$

and thus $\rho A^{K}$ is exponentially stable.

Let $\left(v_{1}, w_{1}, x_{1}, y_{1}\right) \in \mathcal{B}^{S_{1}}$ and $\left(v_{2}, w_{2}, x_{2}, y_{2}\right) \in \mathcal{B}^{S_{2}}$. By Lemma 2.2, it follows that, for $i \in\{1,2\}$,

$$
\begin{aligned}
x_{i}^{+} & =A^{K} x_{i}+B^{K}\left(f\left(y_{i}+w_{i}\right)-K y_{i}\right)+\left(B_{\mathrm{e}}+B^{K} K D_{\mathrm{e}}\right) v_{i}, \\
y_{i} & =C^{K} x_{i}+D^{K}\left(f\left(y_{i}+w_{i}\right)-K y_{i}\right)+(I-D K)^{-1} D_{\mathrm{e}} v_{i} .
\end{aligned}
$$

Forming the differences gives

$$
\begin{aligned}
\left(x_{1}-x_{2}\right)^{+}=A^{K}\left(x_{1}-x_{2}\right)+B^{K}\left(f\left(y_{1}+w_{1}\right)\right. & -f\left(y_{2}+w_{2}\right) \\
& \left.-K\left(y_{1}+w_{1}-y_{2}-w_{2}\right)\right)+\eta, \\
y_{1}-y_{2}=C^{K}\left(x_{1}-x_{2}\right)+D^{K}\left(f\left(y_{1}+w_{1}\right)\right. & -f\left(y_{2}+w_{2}\right) \\
& \left.-K\left(y_{1}+w_{1}-y_{2}-w_{2}\right)\right)+\nu
\end{aligned}
$$

where

$$
\begin{aligned}
& \eta:=\left(B_{\mathrm{e}}+B^{K} K D_{\mathrm{e}}\right)\left(v_{1}-v_{2}\right)+B^{K} K\left(w_{1}-w_{2}\right) \quad \text { and } \\
& \nu:=(I-D K)^{-1} D_{\mathrm{e}}\left(v_{1}-v_{2}\right)+D^{K} K\left(w_{1}-w_{2}\right) .
\end{aligned}
$$

As all the operators involved are bounded, there exists $\kappa>0$ such that

$$
\left\|\left(\begin{array}{c}
\rho \eta(t) \\
\nu(t)
\end{array}\right)\right\|_{X \times Y} \leq \kappa\left\|\left(\begin{array}{c}
\left(v_{1}-v_{2}\right)(t) \\
\left(w_{1}-w_{2}\right)(t)
\end{array}\right)\right\|_{V \times Y}, \quad \forall t \in \mathbb{Z}_{+} .
$$


Defining $\tilde{u}_{\rho}(t)=\rho^{t} \tilde{u}(t)$, for all $t \in \mathbb{Z}_{+}$and all sequences $\tilde{u}$, then (3.9) and (3.10) yield that

$$
\left.\begin{array}{c}
\left(x_{1}-x_{2}\right)_{\rho}^{+}=\rho A^{K}\left(x_{1}-x_{2}\right)_{\rho}+\rho B^{K} g_{\rho}+\rho \eta_{\rho}, \\
\left(y_{1}-y_{2}\right)_{\rho}=C^{K}\left(x_{1}-x_{2}\right)_{\rho}+D^{K} g_{\rho}+\nu_{\rho},
\end{array}\right\}
$$

where

$$
g(t):=f\left(y_{1}(t)+w_{1}(t)\right)-f\left(y_{2}(t)+w_{2}(t)\right)-K\left(y_{1}(t)+w_{1}(t)-y_{2}(t)-w_{2}(t)\right), \quad \forall t \in \mathbb{Z}_{+} .
$$

We thus have that

$$
\left(g_{\rho},\left(\begin{array}{c}
\rho \eta_{\rho} \\
\nu_{\rho}
\end{array}\right),\left(x_{1}-x_{2}\right)_{\rho},\left(y_{1}-y_{2}\right)_{\rho}\right)
$$

is in the behaviour of the linear system $\left(\rho A^{K}, \rho B^{K},\left(\begin{array}{ll}I & 0\end{array}\right), C^{K}, D^{K},\left(\begin{array}{ll}0 & I\end{array}\right)\right) \in \mathbb{L}$ where $V=X \times$ $Y$. Therefore, since $\rho A^{K}$ is exponentially stable, an application of Lemma 2.3 to system (3.12) yields that, for all $t \in \mathbb{Z}_{+}$,

$$
\left\|\pi_{t}\left(y_{1}-y_{2}\right)_{\rho}\right\|_{\ell^{2}} \leq c_{1}\left\|\left(x_{1}-x_{2}\right)_{\rho}(0)\right\|_{X}+\|\mathbf{H}\|_{H^{\infty}}\left\|\pi_{t} g_{\rho}\right\|_{\ell^{2}}+c_{3}\left\|\pi_{t}\left(\begin{array}{c}
\rho \eta_{\rho} \\
\nu_{\rho}
\end{array}\right)\right\|_{\ell^{2}},
$$

where $c_{1}$ and $c_{3}$ are constants independent of $\left(v_{1}, w_{1}, x_{1}, y_{1}\right)$ and $\left(v_{2}, w_{2}, x_{2}, y_{2}\right)$. By definition of $g$ and assumption (3.3), we have, for all $t \in \mathbb{Z}_{+}$,

$$
\begin{aligned}
\left\|g_{\rho}(t)\right\|_{U} & =\rho^{t}\left\|f\left(y_{1}(t)+w_{1}(t)\right)-f\left(y_{2}(t)+w_{2}(t)\right)-K\left(y_{1}(t)+w_{1}(t)-y_{2}(t)-w_{2}(t)\right)\right\|_{U} \\
& \leq(r-\delta) \rho^{t}\left\|\left(y_{1}+w_{1}-y_{2}-w_{2}\right)(t)\right\|_{Y} \\
& =(r-\delta)\left\|\left(y_{1}+w_{1}-y_{2}-w_{2}\right)_{\rho}(t)\right\|_{Y}
\end{aligned}
$$

therefore

$$
\left\|\pi_{t} g_{\rho}\right\|_{\ell^{2}} \leq(r-\delta)\left\|\pi_{t}\left(y_{1}-y_{2}\right)_{\rho}\right\|_{\ell^{2}}+(r-\delta)\left\|\pi_{t}\left(w_{1}-w_{2}\right)_{\rho}\right\|_{\ell^{2}}, \quad \forall t \in \mathbb{Z}_{+} .
$$

Substituting (3.8), (3.11) and (3.14) into (3.13) and rearranging yields

$$
\begin{aligned}
\left\|\pi_{t}\left(y_{1}-y_{2}\right)\right\|_{\ell_{\rho}^{2}} & =\left\|\pi_{t}\left(y_{1}-y_{2}\right)_{\rho}\right\|_{\ell^{2}} \\
& \leq c_{4}\left\|\left(x_{1}-x_{2}\right)_{\rho}(0)\right\|_{X}+c_{5}\left\|\pi_{t}\left(\begin{array}{c}
\left(v_{1}-v_{2}\right)_{\rho} \\
\left(w_{1}-w_{2}\right)_{\rho}
\end{array}\right)\right\|_{\ell^{2}}, \quad \forall t \in \mathbb{Z}_{+} .
\end{aligned}
$$

where

$$
c_{4}:=\frac{c_{1}}{1-(r-\delta)\|\mathbf{H}\|_{H^{\infty}}}, \quad c_{5}:=\frac{\kappa c_{3}+(r-\delta)\|\mathbf{H}\|_{H^{\infty}}}{1-(r-\delta)\|\mathbf{H}\|_{H^{\infty}}} .
$$

Inequality (3.5) now follows from (3.15) with $b:=\max \left\{c_{4}, c_{5}\right\}$. Similarly, by another application of Lemma 2.3 to (3.12), we obtain that

$$
\left\|\pi_{t}\left(x_{1}-x_{2}\right)_{\rho}\right\|_{\ell^{2}} \leq c_{1}\left\|\left(x_{1}-x_{2}\right)(0)\right\|_{X}+c_{2}\left\|\pi_{t-1} g_{\rho}\right\|_{\ell^{2}}+c_{3}\left\|\pi_{t-1}\left(\begin{array}{c}
\rho \eta_{\rho} \\
\nu_{\rho}
\end{array}\right)\right\|_{\ell^{2}}, \quad \forall t \in \mathbb{N} .
$$

Substituting (3.14) and (3.15) into the above, we see that, for all $t \in \mathbb{N}$,

$$
\begin{aligned}
& \left\|\pi_{t}\left(x_{1}-x_{2}\right)\right\|_{\ell_{\rho}^{2}}=\left\|\pi_{t}\left(x_{1}-x_{2}\right)_{\rho}\right\|_{\ell^{2}} \\
& \leq c_{1}\left\|\left(x_{1}-x_{2}\right)(0)\right\|_{X}+c_{2}(r-\delta)\left(\left\|\pi_{t-1}\left(y_{1}-y_{2}\right)_{\rho}\right\|_{\ell^{2}}+\left\|\pi_{t-1}\left(w_{1}-w_{2}\right)_{\rho}\right\|_{\ell^{2}}\right)+c_{3}\left\|\pi_{t-1}\left(\begin{array}{c}
\rho \eta_{\rho} \\
\nu_{\rho}
\end{array}\right)\right\|_{\ell^{2}} \\
& \leq c_{6}\left\|\left(x_{1}-x_{2}\right)(0)\right\|_{X}+c_{7}\left\|\pi_{t-1}\left(\begin{array}{c}
\left(v_{1}-v_{2}\right)_{\rho} \\
\left(w_{1}-w_{2}\right)_{\rho}
\end{array}\right)\right\|_{\ell^{2}},
\end{aligned}
$$


where $c_{6}:=c_{1}+c_{2}(r-\delta) c_{4}$ and $c_{7}:=c_{3} \kappa+c_{2}(r-\delta)\left(c_{5}+1\right)$. Setting $a:=\max \left\{c_{6}, c_{7}\right\}$ in the above inequality gives (3.4), proving statement (i).

We proceed to prove statement (ii). Since $\rho A^{K}$ is exponentially stable, we have that

$$
c_{8}:=\sup _{t \in \mathbb{Z}_{+}}\left\|\left(\rho A^{K}\right)^{t}\right\|<\infty .
$$

Applying the variation-of-parameters formula to (3.12) gives

$$
\begin{aligned}
\left(x_{1}-x_{2}\right)_{\rho}(t)= & \left(\rho A^{K}\right)^{t}\left(x_{1}-x_{2}\right)(0)+\sum_{k=0}^{t-1}\left(\rho A^{K}\right)^{t-1-k} \rho B^{K} g_{\rho}(k) \\
& +\sum_{k=0}^{t-1}\left(\rho A^{K}\right)^{t-1-k} \rho \eta_{\rho}(k), \quad \forall t \in \mathbb{N} .
\end{aligned}
$$

An application of the triangle and Hölder's inequalities yield, for all $t \in \mathbb{N}$,

$$
\begin{aligned}
\left\|\left(x_{1}-x_{2}\right)_{\rho}(t)\right\|_{X} \leq & c_{8}\left\|\left(x_{1}-x_{2}\right)(0)\right\|_{X}+\left\|\rho B^{K}\right\| \sum_{k=0}^{t-1}\left\|\left(\rho A^{K}\right)^{t-1-k}\right\|\left\|g_{\rho}(k)\right\|_{U} \\
& +\rho \sum_{k=0}^{t-1}\left\|\left(\rho A^{K}\right)^{t-1-k}\right\|\left\|\eta_{\rho}(k)\right\|_{U} \\
\leq & c_{8}\left\|\left(x_{1}-x_{2}\right)(0)\right\|_{X}+\left\|\rho B^{K}\right\|\left(\sum_{k=0}^{t-1}\left\|\left(\rho A^{K}\right)^{k}\right\|^{2}\right)^{1 / 2}\left\|\pi_{t-1} g_{\rho}\right\|_{\ell^{2}} \\
& +\rho\left(\sum_{k=0}^{t-1}\left\|\left(\rho A^{K}\right)^{k}\right\|^{2}\right)^{1 / 2}\left\|\pi_{t-1} \eta_{\rho}\right\|_{\ell^{2}} .
\end{aligned}
$$

Substituting (3.14), and then (3.15) and (3.11), into the above estimate gives

$$
\begin{aligned}
& \left\|\left(x_{1}-x_{2}\right)_{\rho}(t)\right\|_{X} \leq c_{8}\left\|\left(x_{1}-x_{2}\right)(0)\right\|_{X}+\rho\left(\sum_{k=0}^{t-1}\left\|\left(\rho A^{K}\right)^{k}\right\|^{2}\right)^{1 / 2}\left\|\pi_{t-1} \eta_{\rho}\right\|_{\ell^{2}} \\
& \quad+(r-\delta)\left\|\rho B^{K}\right\|\left(\sum_{k=0}^{t-1}\left\|\left(\rho A^{K}\right)^{k}\right\|^{2}\right)^{1 / 2}\left(\left\|\pi_{t-1}\left(y_{1}-y_{2}\right)_{\rho}\right\|_{\ell^{2}}+\left\|\pi_{t}\left(w_{1}-w_{2}\right)_{\rho}\right\|_{\ell^{2}}\right) \\
& \leq c_{9}\left\|\left(x_{1}-x_{2}\right)(0)\right\|_{X}+c_{10}\left\|\pi_{t-1}\left(\begin{array}{c}
\left(v_{1}-v_{2}\right)_{\rho} \\
\left(w_{1}-w_{2}\right)_{\rho}
\end{array}\right)\right\|_{\ell^{2}}, \quad \forall t \in \mathbb{N}
\end{aligned}
$$

where

$$
c_{9}:=c_{8}+c_{4}(r-\delta)\left\|\rho B^{K}\right\|\left(\sum_{k=0}^{\infty}\left\|\left(\rho A^{K}\right)^{k}\right\|^{2}\right)^{1 / 2}
$$

and

$$
c_{10}:=\kappa\left(\sum_{k=0}^{\infty}\left\|\left(\rho A^{K}\right)^{k}\right\|^{2}\right)^{1 / 2}+\left(c_{5}+1\right)(r-\delta)\left\|\rho B^{K}\right\|\left(\sum_{k=0}^{\infty}\left\|\left(\rho A^{K}\right)^{k}\right\|^{2}\right)^{1 / 2} .
$$

Estimate (3.16) can be written as

$$
\left\|\left(x_{1}-x_{2}\right)(t)\right\|_{X} \leq c_{9} \rho^{-t}\left\|\left(x_{1}-x_{2}\right)(0)\right\|_{X}+c_{10} \rho^{-t}\left\|\pi_{t-1}\left(\begin{array}{c}
\left(v_{1}-v_{2}\right)_{\rho} \\
\left(w_{1}-w_{2}\right)_{\rho}
\end{array}\right)\right\|_{\ell^{2}}, \quad \forall t \in \mathbb{N} .
$$


If $q \in(2, \infty)$, then there exists $p \in(1, \infty)$ such that $2 / q+1 / p=1$ and, using Hölder's inequality again,

$$
\begin{aligned}
\left\|\pi_{t}\left(\begin{array}{c}
\left(v_{1}-v_{2}\right)_{\rho} \\
\left(w_{1}-w_{2}\right)_{\rho}
\end{array}\right)\right\|_{\ell^{2}}^{2} & =\sum_{k=0}^{t} \rho^{2 k}\left\|\left(\begin{array}{c}
\left(v_{1}-v_{2}\right)(k) \\
\left(w_{1}-w_{2}\right)(k)
\end{array}\right)\right\|_{V \times Y}^{2} \leq\left(\sum_{k=0}^{t} \rho^{2 k p}\right)^{1 / p}\left\|\pi_{t}\left(\begin{array}{c}
v_{1}-v_{2} \\
w_{1}-w_{2}
\end{array}\right)\right\|_{\ell^{q}}^{2} \\
& =\left(\frac{\rho^{2 p(t+1)}-1}{\rho^{2 p}-1}\right)^{1 / p}\left\|\pi_{t}\left(\begin{array}{c}
v_{1}-v_{2} \\
w_{1}-w_{2}
\end{array}\right)\right\|_{\ell^{q}}^{2} \\
& \leq \rho^{2 t}\left(\frac{\rho^{2}}{\left(\rho^{2 p}-1\right)^{1 / p}}\right)\left\|\pi_{t}\left(\begin{array}{c}
v_{1}-v_{2} \\
w_{1}-w_{2}
\end{array}\right)\right\|_{\ell^{q}}^{2}, \quad \forall t \in \mathbb{Z}_{+} .
\end{aligned}
$$

If $q=2$, then since $\rho>1$,

$$
\begin{aligned}
\left\|\pi_{t}\left(\begin{array}{c}
\left(v_{1}-v_{2}\right)_{\rho} \\
\left(w_{1}-w_{2}\right)_{\rho}
\end{array}\right)\right\|_{\ell^{2}} & =\left(\sum_{k=0}^{t} \rho^{2 k}\left\|\left(\begin{array}{c}
\left(v_{1}-v_{2}\right)(k) \\
\left(w_{1}-w_{2}\right)(k)
\end{array}\right)\right\|_{V \times Y}^{2}\right)^{1 / 2} \\
& \leq \rho^{t}\left\|\pi_{t}\left(\begin{array}{c}
v_{1}-v_{2} \\
w_{1}-w_{2}
\end{array}\right)\right\|_{\ell^{2}}, \quad \forall t \in \mathbb{Z}_{+} .
\end{aligned}
$$

Finally, if $q=\infty$,

$$
\begin{aligned}
& \left\|\pi_{t}\left(\begin{array}{c}
\left(v_{1}-v_{2}\right)_{\rho} \\
\left(w_{1}-w_{2}\right)_{\rho}
\end{array}\right)\right\|_{\ell^{2}}=\left(\sum_{k=0}^{t} \rho^{2 k}\left\|\left(\begin{array}{c}
\left(v_{1}-v_{2}\right)(k) \\
\left(w_{1}-w_{2}\right)(k)
\end{array}\right)\right\|_{V \times Y}^{2}\right)^{1 / 2} \\
& \leq\left(\sum_{k=0}^{t} \rho^{2 k}\right)^{1 / 2} \max _{s \in \underline{t}}\left\|\left(\begin{array}{c}
\left(v_{1}-v_{2}\right)(s) \\
\left(w_{1}-w_{2}\right)(s)
\end{array}\right)\right\|_{V \times Y} \\
& =\left(\frac{\rho^{2(t+1)}-1}{\rho^{2}-1}\right)^{1 / 2} \max _{s \in \underline{t}}\left\|\left(\begin{array}{c}
\left(v_{1}-v_{2}\right)(s) \\
\left(w_{1}-w_{2}\right)(s)
\end{array}\right)\right\|_{V \times Y} \\
& \leq \rho^{t}\left(\frac{\rho}{\left(\rho^{2}-1\right)^{1 / 2}}\right)\left\|\pi_{t}\left(\begin{array}{c}
v_{1}-v_{2} \\
w_{1}-w_{2}
\end{array}\right)\right\|_{\ell \infty}, \quad \forall t \in \mathbb{Z}_{+} .
\end{aligned}
$$

Therefore, for every $q \in[2, \infty]$, there exists a positive constant $c_{11}$ such that

$$
\left\|\pi_{t}\left(\begin{array}{c}
\left(v_{1}-v_{2}\right)_{\rho} \\
\left(w_{1}-w_{2}\right)_{\rho}
\end{array}\right)\right\|_{\ell^{2}} \leq \rho^{t} c_{11}\left\|\pi_{t}\left(\begin{array}{c}
\left(v_{1}-v_{2}\right)_{\rho} \\
\left(w_{1}-w_{2}\right)_{\rho}
\end{array}\right)\right\|_{\ell^{q}}, \quad \forall t \in \mathbb{Z}_{+},
$$

which, when substituted into (3.17) gives that, for any $q \in[2, \infty]$,

$$
\begin{aligned}
\left\|\left(x_{1}-x_{2}\right)(t)\right\|_{X} & \leq c_{9} \rho^{-t}\left\|\left(x_{1}-x_{2}\right)(0)\right\|_{X}+c_{10} \rho^{-1} c_{11}\left\|\pi_{t-1}\left(\begin{array}{c}
\left(v_{1}-v_{2}\right)_{\rho} \\
\left(w_{1}-w_{2}\right)_{\rho}
\end{array}\right)\right\|_{\ell^{q}} \\
& \leq c_{9} \rho^{-t}\left\|\left(x_{1}-x_{2}\right)(0)\right\|_{X}+c_{10} c_{11}\left\|\pi_{t-1}\left(\begin{array}{c}
\left(v_{1}-v_{2}\right)_{\rho} \\
\left(w_{1}-w_{2}\right)_{\rho}
\end{array}\right)\right\|_{\ell^{q}}, \quad \forall t \in \mathbb{N} .
\end{aligned}
$$

Setting $c:=\max \left\{c_{9}, c_{10} c_{11}\right\}$ and $\theta=\rho^{-1}$ gives (3.6).

It remains to establish (3.7). We note that,

$$
\left\|D^{K}\right\| \leq\left\|\mathbf{G}^{K}\right\|_{H^{\infty}} \leq \frac{1}{r}<\frac{1}{r-\delta},
$$

whence, appealing to (3.3) and (3.10),

$$
\begin{gathered}
\left\|\left(y_{1}-y_{2}\right)(t)\right\|_{Y} \leq\left\|C^{K}\right\|\left\|\left(x_{1}-x_{2}\right)(t)\right\|_{X}+\left\|D^{K}\right\|(r-\delta)\left(\left\|\left(y_{1}-y_{2}\right)(t)\right\|_{Y}+\left\|\left(w_{1}-w_{2}\right)(t)\right\|_{Y}\right) \\
+\|\nu(t)\|_{Y} \quad \forall t \in \mathbb{Z}_{+} .
\end{gathered}
$$


Define

$$
d_{1}:=\frac{1}{1-(r-\delta)\left\|D^{K}\right\|},
$$

which is positive by (3.18). Substituting (3.6) into (3.19) and setting $d_{2}:=d_{1}\left\|C^{K}\right\| c$ and $d_{3}:=d_{1}\left(\left\|C^{K}\right\| c+\kappa+(r-\delta)\left\|D^{K}\right\|\right)$, we see that

$$
\left\|\left(y_{1}-y_{2}\right)(t)\right\|_{Y} \leq d_{2} \theta^{t}\left\|\left(x_{1}-x_{2}\right)(0)\right\|_{X}+d_{3}\left\|\pi_{t}\left(\begin{array}{c}
\left(v_{1}-v_{2}\right)_{\rho} \\
\left(w_{1}-w_{2}\right)_{\rho}
\end{array}\right)\right\|_{\ell^{q}} \quad \forall t \in \mathbb{Z}_{+} .
$$

Setting $d:=\max \left\{d_{2}, d_{3}\right\}$ completes the proof.

Remark 3.4. (a) By inspecting the above proof, we are able to see that Theorem 3.2 holds true if $X$ and $V$ are real Banach spaces and $Y$ and $U$ are real Hilbert spaces, provided that the complex ball condition $\mathbb{B}_{\mathrm{c}}(K, r) \subseteq \mathbb{S}_{\mathrm{c}}(\mathbf{G})$ holds, with $\mathbb{B}_{\mathrm{c}}(K, r):=\{M \in$ $\left.\mathcal{L}\left(Y_{\mathrm{c}}, U_{\mathrm{c}}\right):\|M-K\|<r\right\}$ and $\mathbb{S}_{\mathrm{c}}(\mathbf{G}):=\left\{M \in \mathcal{L}\left(Y_{\mathrm{c}}, U_{\mathrm{c}}\right): I-D M\right.$ is invertible and $\mathbf{G}^{M} \in$ $\left.H^{\infty}\left(\mathcal{L}\left(U_{\mathrm{c}}, Y_{\mathrm{c}}\right)\right)\right\}$, where $Y_{\mathrm{c}}$ and $U_{\mathrm{c}}$ denote the complexifications of $Y$ and $U$, respectively. The same can be said of the rest of the results in sections $3,4,6$ and 5 .

(b) For later purposes, it will be useful to consider Theorem 3.2 in the (rather degenerate) situation wherein $\mathbf{G}=0$. If $\mathbf{G}=0$, then $\mathbf{G}^{K}=0$ for all $K \in \mathcal{L}(Y, U)$ and by Lemma 2.1, it follows that $\mathbb{B}(K, r) \subseteq \mathbb{S}(\mathbf{G})$ for all $r>0$. Consequently, in the case wherein $\mathbf{G}=0$, the conclusions of Theorem 3.2 hold, provided that there exists $K \in \mathcal{L}(Y, U)$ such that

$$
\sup _{(\xi, \zeta) \in S_{1} \times S_{2}, \xi \neq \zeta} \frac{\|f(\xi)-f(\zeta)-K(\xi-\zeta)\|_{U}}{\|\xi-\zeta\|_{Y}}<\infty .
$$

We next present a corollary to Theorem 3.2 which is reminiscent of the circle criterion. To this end, for $\alpha \in(0,1]$, we denote by $H_{\alpha}^{*}(\mathcal{L}(U, Y))$ the set of functions $\mathbf{H}: \mathbb{E}_{\alpha} \rightarrow \mathcal{L}(U, Y)$ which are holomorphic on $\mathbb{E}_{\alpha}$, with the exception of isolated singularities, that is, poles and essential singularities. We always assume that removable singularities have been removed via holomorphic extension. For convenience, we set $H^{*}(\mathcal{L}(U, Y)):=H_{1}^{*}(\mathcal{L}(U, Y))$.

Let $\mathbf{H} \in H_{\alpha}^{*}(\mathcal{L}(U))$. We define $\Sigma_{\mathbf{H}} \subseteq \mathbb{E}_{\alpha}$ to be the set of isolated singularities of $\mathbf{H}$. The function $\mathbf{H}$ is said to be positive real if

$$
\operatorname{Re}\langle\mathbf{H}(z) u, u\rangle_{U} \geq 0, \quad \forall u \in U, \forall z \in \mathbb{E} \backslash \Sigma_{\mathbf{H}} .
$$

The following lemma is an analogue to [15, Proposition 3.3], which concerns positive real functions on the right-half complex plane, for positive real functions on the exterior of the unit disc. We relegate the proof to Appendix A.

Lemma 3.5. Let $\mathbf{H} \in H^{*}(\mathcal{L}(U))$ be positive real. Then $\mathbf{H}$ does not have any singularities in $\mathbb{E} \cup\{\infty\}$.

We shall also require the following technical lemma, the proof of which is identical, mutatis mutandis, to that of [15, Corollary 2.3].

Lemma 3.6. Let $\mathbf{H} \in H^{*}(\mathcal{L}(U))$ be positive real. Then, $I+\mathbf{H}(z)$ is invertible for every $z \in \mathbb{E}$ and

$$
\left\|(I-\mathbf{H})(I+\mathbf{H})^{-1}\right\|_{H^{\infty}} \leq 1 .
$$


Corollary 3.7. Let $\Sigma \in \mathbb{L}$ be stabilisable and detectable, $S \subseteq Y$ non-empty and $K_{1}, K_{2} \in$ $\mathcal{L}(Y, U)$ with $K_{1} \in \mathbb{A}(D)$. If $\left(I-K_{2} \mathbf{G}\right)\left(I-K_{1} \mathbf{G}\right)^{-1}$ is positive real and there exists $\varepsilon>0$ such that

$$
\operatorname{Re}\left\langle f(\zeta+\xi)-f(\xi)-K_{1} \zeta, f(\zeta+\xi)-f(\xi)-K_{2} \zeta\right\rangle_{U} \leq-\varepsilon\|\zeta\|_{Y}^{2}, \quad \forall \zeta \in Y, \forall \xi \in S,
$$

then statements (i) and (ii) of Theorem 3.2 with $S_{1}=Y, S_{2}=S$ hold.

The following proof is in part inspired by a method outlined in the proof of [15, Theorem 6.8$]$.

Proof. We define

$$
L:=\left(K_{1}-K_{2}\right) / 2, \quad M:=\left(K_{1}+K_{2}\right) / 2,
$$

and rewrite (3.21) so that

$$
\begin{aligned}
-\varepsilon\|\zeta\|_{Y}^{2} & \geq \operatorname{Re}\langle f(\zeta+\xi)-f(\xi)-(L+M) \zeta, f(\zeta+\xi)-f(\xi)+(L-M) \zeta\rangle \\
& =-\|L \zeta\|_{U}^{2}+\|f(\zeta+\xi)-f(\xi)-M \zeta\|_{U}^{2}, \quad \forall \zeta \in Y, \forall \xi \in S .
\end{aligned}
$$

We deduce from (3.22) that $\|L \zeta\|_{U} \geq \sqrt{\varepsilon}\|\zeta\|_{Y}$, for all $\zeta \in Y$, which in turn implies that

$$
\left\|L^{*} L \zeta\right\|_{Y}\|\zeta\|_{Y} \geq\left|\left\langle L^{*} L \zeta, \zeta\right\rangle\right|=\|L \zeta\|_{U}^{2} \geq \varepsilon\|\zeta\|_{Y}^{2} \quad \forall \zeta \in Y .
$$

Hence $L^{*} L$ is bounded away from 0 and, by combining this with the self-adjointness of $L^{*} L$, we have that $L^{*} L$ is invertible. We define $L^{\#}:=\left(L^{*} L\right)^{-1} L^{*}$ and let $Q:=L L^{\#}$. It is clear that $Q^{2}=L L^{\#} L L^{\#}=Q$ and, since $L$ has a left inverse, im $L$ is closed. Thus,

$$
\operatorname{im} L=\left(\operatorname{ker} L^{*}\right)^{\perp}=\left(\operatorname{ker} L^{\#}\right)^{\perp} .
$$

Therefore, $Q$ is the orthogonal projection onto $\left(\operatorname{ker} L^{\#}\right)^{\perp}$ along $\operatorname{ker} L^{\#}$. Utilising this with (3.22) gives that

$$
\begin{aligned}
\left\|\left(f \circ L^{\#}\right)(\zeta+\xi)-\left(f \circ L^{\#}\right)(\xi)-M L^{\#} \zeta\right\|_{U}^{2} & \leq\|Q \zeta\|_{U}^{2}-\varepsilon\left\|L^{\#} \zeta\right\|_{Y}^{2} \\
& \leq\|\zeta\|_{Y}^{2}-\varepsilon\left\|L^{\#} \zeta\right\|_{Y}^{2}, \forall \zeta \in Y, \forall \xi \in S .
\end{aligned}
$$

Moreover, since $L^{\#}$ is bounded away from 0 on $\operatorname{im} L$, there exists $\nu>0$ such that

$$
\left\|L^{\#} \zeta\right\|_{Y} \geq \nu\|\zeta\|_{Y}, \quad \forall \zeta \in \operatorname{im} L .
$$

Hence, combining this with (3.23) yields

$$
\begin{aligned}
\left\|\left(f \circ L^{\#}\right)(\zeta+\xi)-\left(f \circ L^{\#}\right)(\xi)-M L^{\#} \zeta\right\|_{U}^{2} & =\left\|\left(f \circ L^{\#}\right)(Q \zeta+Q \xi)-\left(f \circ L^{\#}\right)(Q \xi)-M L^{\#} Q \zeta\right\|_{U}^{2} \\
& \leq\|Q \zeta\|_{U}^{2}-\varepsilon \nu^{2}\|Q \zeta\|_{U}^{2}, \quad \forall \zeta \in Y, \forall \xi \in S,
\end{aligned}
$$

and so

$$
\left\|\left(f \circ L^{\#}\right)(\zeta+\xi)-\left(f \circ L^{\#}\right)(\xi)-M L^{\#} \zeta\right\|_{U} \leq \sqrt{1-\varepsilon \nu^{2}}\|\zeta\|_{Y}, \quad \forall \zeta \in Y, \forall \xi \in S .
$$

Next, on the one hand we compute that

$$
\left(I-K_{2} \mathbf{G}\right)\left(I-K_{1} \mathbf{G}\right)^{-1}=\left(I-K_{1} \mathbf{G}+2 L \mathbf{G}\right)\left(I-K_{1} \mathbf{G}\right)^{-1}=I+2 L \mathbf{G}\left(I-K_{1} \mathbf{G}\right)^{-1},
$$

and, on the other, that

$$
\left(I-K_{2} \mathbf{G}\right)\left(I-K_{1} \mathbf{G}\right)^{-1}=\left(I-2 M \mathbf{G}+K_{1} \mathbf{G}\right)\left(I-K_{1} \mathbf{G}\right)^{-1} .
$$


Invoking the positive realness of $\left(I-K_{2} \mathbf{G}\right)\left(I-K_{1} \mathbf{G}\right)^{-1}$ along with Lemma 3.6, employing the expressions (3.25) and (3.26) yields that

$$
\begin{aligned}
1 & \geq\left\|2 L \mathbf{G}\left(I-K_{1} \mathbf{G}\right)^{-1}\left(I+\left(I-2 M \mathbf{G}+K_{1} \mathbf{G}\right)\left(I-K_{1} \mathbf{G}\right)^{-1}\right)^{-1}\right\| \\
& =\left\|2 L \mathbf{G}(2 I-2 M \mathbf{G})^{-1}\right\| \\
& =\left\|L \mathbf{G}(I-M \mathbf{G})^{-1}\right\| .
\end{aligned}
$$

In addition, evidently

$$
L \mathbf{G}(I-M \mathbf{G})^{-1}=L \mathbf{G}\left(I-M L^{\#} L \mathbf{G}\right)^{-1}=(L \mathbf{G})^{M L^{\#}},
$$

and thus, by Lemma 2.1, we see that

$$
\mathbb{B}\left(M L^{\#}, 1\right) \subseteq \mathbb{S}(L \mathbf{G}) .
$$

Finally, let $(v, w, x, y) \in \mathcal{B}$ and note that, since $I=L^{\#} L$,

$$
\begin{aligned}
& x^{+}=A x+B\left(f \circ L^{\#}\right)(L y+L w)+B_{\mathrm{e}} v, \\
& L y=L C x+L D\left(f \circ L^{\#}\right)(L y+L w)+L D_{\mathrm{e}} v,
\end{aligned}
$$

which shows that $(v, L w, x, L y)$ satisfies $(2.3)$ with non-linearity $f \circ L^{\#}$ and linear component given by $\left(A, B, B_{\mathrm{e}}, L C, L D, L D_{\mathrm{e}}\right)$. Moreover, since $\Sigma$ is stabilisable and detectable, it is clear, by the left invertibility of $L$, that $\left(A, B, B_{\mathrm{e}}, L C, L D, L D_{\mathrm{e}}\right)$ is also stabilisable and detectable. Combining this with (3.24) and (3.27), we see that the hypotheses of Theorem 3.2 are satisfied, and so the left-invertibility of $L$ completes the proof.

Remark 3.8. An inspection of the proof of Corollary 3.7 shows that if the estimate given in (3.21) instead holds for all $\zeta \in S_{1}$ and for all $\xi \in S$, where $S_{1}$ is a non-empty subset of $Y$, then the conclusions of Corollary 3.7 remain valid, provided that $K_{1}-K_{2}$ is left invertible.

\section{Convergence properties}

In this section we use Theorem 3.2 to establish convergence properties of the state $x$ and output $y$ of the Lur'e system (2.3) when subject to converging or periodic inputs $v$ and $w$.

\subsection{The converging-input converging-state property}

Here we give conditions under which the Lur'e system exhibits the so-called converging-input converging-state property. We say that, for $\Sigma \in \mathbb{L}$ and $f: Y \rightarrow U$, the discrete-time Lur'e system (2.3) has the converging-input converging-state (CICS) property if, for every $v^{\infty} \in V$ and $w^{\infty} \in Y$, there exists $x^{\infty} \in X$ such that, for every $(v, w, x, y) \in \mathcal{B}$ with $\lim _{t \rightarrow \infty} v(t)=v^{\infty}$ and $\lim _{t \rightarrow \infty} w(t)=w^{\infty}$, we have that $\lim _{t \rightarrow \infty} x(t)=x^{\infty}$. We note that some authors (see, for example, [47]) use the term CICS for the special case wherein $v^{\infty}=0, w^{\infty}=0$ and $x^{\infty}=0$.

Our main result of this section is Theorem 4.3, from which we obtain sufficient conditions for the CICS property in Corollary 4.6. We comment that if $w$ in (2.3) is perceived to be an output disturbance to the system, then convergence of $w$ is not an assumption which will be generically satisfied. Hence, in addition to considering the CICS property, we also develop a result for bounded but not necessarily convergent $w$, see Corollary 4.7 .

Let $K \in \mathbb{S}(\mathbf{G})$ and define the map

$$
F_{K}: Y \rightarrow Y, \quad \xi \mapsto \xi-\mathbf{G}^{K}(1)(f(\xi)-K \xi) .
$$


For ease of notation in the sequel, for given $\xi \in Y$, we write $F_{K}^{-1}(\xi)$ to denote the inverse image of the singleton $\{\xi\}$ under $F_{K}$, instead of the more cumbersome $F_{K}^{-1}(\{\xi\})$. Moreover, we denote the cardinality of $F_{K}^{-1}(\xi)$ by $\# F_{K}^{-1}(\xi)$.

To facilitate the proofs of the main results in this section, it is useful to state two lemmas, the proofs of which may be found in Appendix A.

Lemma 4.1. Let $\Sigma \in \mathbb{L}, S \subseteq Y$ be non-empty, $K \in \mathbb{S}(\mathbf{G}), F_{K}$ be given by (4.1), and assume that $\gamma:=1 /\left\|\mathbf{G}^{K}\right\|_{H^{\infty}}<\infty$ and

$$
\|f(\zeta+\xi)-f(\xi)-K \zeta\|_{U}<\gamma\|\zeta\|_{Y}, \quad \forall \zeta \in Y \backslash\{0\}, \forall \xi \in S .
$$

Then the following statements hold.

(i) $\# F_{K}^{-1}(\xi)=1$, for all $\xi \in Y$ such that $F_{K}^{-1}(\xi) \cap S \neq \emptyset$.

If there exists $\delta>0$ such that $f$ and $K$ satisfy (3.3) with $r=\gamma$ and $S_{1}=S_{2}=Y$, then

(ii) $F_{K}$ is globally Lipschitz and bijective;

(iii) the inverse $F_{K}^{-1}$ is globally Lipschitz.

Although we assume that $\gamma<\infty$ in Lemma 4.1 , if actually $\left\|\mathbf{G}^{K}\right\|_{H^{\infty}}=0$, then $F_{K}$ is the identity map which is trivially globally Lipschitz and bijective.

Lemma 4.2. Let $\Sigma \in \mathbb{L}$ be stabilisable and detectable, $v^{\infty} \in V$ and $w^{\infty} \in Y$. Assume that $K \in \mathbb{S}(\mathbf{G})$ and

$$
\mathcal{T}_{K}:=F_{K}^{-1}\left(C^{K}\left(I-A^{K}\right)^{-1}\left(B_{\mathrm{e}}+B^{K} K D_{\mathrm{e}}\right) v^{\infty}+(I-D K)^{-1} D_{\mathrm{e}} v^{\infty}+\left(I+\mathbf{G}^{K}(1) K\right) w^{\infty}\right)
$$

is nonempty. Let $z^{\infty} \in \mathcal{T}_{K}$ and define $y^{\infty}:=z^{\infty}-w^{\infty}$ and

$$
x^{\infty}:=\left(I-A^{K}\right)^{-1}\left(B^{K}\left(f\left(z^{\infty}\right)-K\left(y^{\infty}\right)\right)+\left(B_{\mathrm{e}}+B^{K} K D_{\mathrm{e}}\right) v^{\infty}\right) .
$$

Then

$$
y^{\infty}=C^{K} x^{\infty}+D^{K}\left(f\left(z^{\infty}\right)-K\left(y^{\infty}\right)\right)+(I-D K)^{-1} D_{\mathrm{e}} v^{\infty},
$$

and $\left(v^{\infty}, w^{\infty}, x^{\infty}, y^{\infty}\right)$ is an equilibrium quadruple of the Lur'e system (2.3).

The formulae in (4.3), (4.4) and (4.5) are motivated by the desire to solve the steady-state equations

$$
x^{\infty}=A x^{\infty}+B f\left(y^{\infty}+w^{\infty}\right)+B_{\mathrm{e}} v^{\infty} \quad \text { and } \quad y^{\infty}=C x^{\infty}+D f\left(y^{\infty}+w^{\infty}\right)+D_{\mathrm{e}} v^{\infty},
$$

where $v^{\infty}$ and $w^{\infty}$ are given, for $x^{\infty}$ and $y^{\infty}$ to yield an equilibrium quadruple $\left(v^{\infty}, w^{\infty}, x^{\infty}, y^{\infty}\right)$. However, $I-A$ need not be invertible, and so G(1) not well defined, hence the inclusion of the loop-shifting term $K$. In the simple case wherein $U=V, B_{\mathrm{e}}=B, D_{\mathrm{e}}=D, K=0$ and $w^{\infty}=0$, the condition that $F_{K}^{-1}\left(\mathbf{G}(1) v^{\infty}\right)$ is nonempty is equivalent to the existence of $y^{\infty} \in Y$ such that

$$
y^{\infty}-\mathbf{G}(1) f\left(y^{\infty}\right)=\mathbf{G}(1) v^{\infty},
$$

and, in this case, (4.4) and (4.5) respectively read

$$
x^{\infty}:=(I-A)^{-1} B\left(f\left(y^{\infty}\right)+v^{\infty}\right) \quad \text { and } \quad y^{\infty}=C x^{\infty}+D f\left(y^{\infty}\right)+D v^{\infty} .
$$

We now state the main theorem in this section. 
Theorem 4.3. Let $\Sigma \in \mathbb{L}$ be stabilisable and detectable, $S \subseteq Y$ be non-empty, $K \in \mathbb{S}(\mathbf{G})$, $v^{\infty} \in V$ and $w^{\infty} \in Y$. Furthermore, assume that $\mathcal{T}_{K} \cap S \neq \emptyset$, where $\mathcal{T}_{K}$ is given by (4.3). If $\gamma:=1 /\left\|\mathbf{G}^{K}\right\|_{H^{\infty}}<\infty$ and there exists $\delta \in(0, \gamma)$ such that (3.3) holds with $r=\gamma, S_{1}=Y$ and $S_{2}=S$, then $\# \mathcal{T}_{K}=1$ and writing $y^{\infty}:=z^{\infty}-w^{\infty}$, where $z^{\infty} \in \mathcal{T}_{K}$, there exist $c>0$ and $a \in(0,1)$ such that for all $(v, w, x, y) \in \mathcal{B}$ and all $t \in \mathbb{Z}_{+}$, we have

$$
\begin{aligned}
& \left\|x(t)-x^{\infty}\right\|_{X}+\left\|y(t)-y^{\infty}\right\|_{Y} \leq c\left(a^{t}\left\|x(0)-x^{\infty}\right\|_{X}+a^{\lceil t / 2\rceil} \max _{s \in \underline{\underline{t t} / 2\rfloor}}\left\|\left(\begin{array}{c}
v(s) \\
w(s)
\end{array}\right)-\left(\begin{array}{c}
v^{\infty} \\
w^{\infty}
\end{array}\right)\right\|_{V \times Y}\right. \\
& \left.+\max _{s \in \underline{\lceil t / 2\rceil}}\left\|\left(\begin{array}{c}
\left(\Lambda_{\lfloor t / 2\rfloor} v\right)(s) \\
\left(\Lambda_{\lfloor t / 2\rfloor} w\right)(s)
\end{array}\right)-\left(\begin{array}{c}
v^{\infty} \\
w^{\infty}
\end{array}\right)\right\|_{V \times Y}\right) .
\end{aligned}
$$

Here $x^{\infty}$ is as in (4.4) and $\left(v^{\infty}, w^{\infty}, x^{\infty}, y^{\infty}\right)$ is an equilibrium quadruple of (2.3). In particular, for all $(v, w, x, y) \in \mathcal{B}$ with $\lim _{t \rightarrow \infty} v(t)=v^{\infty}$ and $\lim _{t \rightarrow \infty} w(t)=w^{\infty}$, we have $\lim _{t \rightarrow \infty} x(t)=$ $x^{\infty}$ and $\lim _{t \rightarrow \infty} y(t)=y^{\infty}$.

Before proving Theorem 4.3, we provide some commentary.

Remark 4.4. (a) Under the hypotheses of Theorem 4.3, we note that $x^{\infty}$ and $y^{\infty}$ given by (4.4) and (4.5) do not depend on the choice of $K$. Indeed, if $K_{1}, K_{2} \in \mathbb{S}(\mathbf{G}), v^{\infty} \in V$, $w^{\infty} \in Y, \Sigma$ is stabilisable and detectable and (3.3) holds for each $K_{l}$ and $\gamma_{l}:=1 /\left\|\mathbf{G}^{K_{l}}\right\|_{H^{\infty}}$, where $l \in\{1,2\}$, then Theorem 4.3 guarantees that $x_{1}^{\infty}=x_{2}^{\infty}$ and $y_{1}^{\infty}=y_{2}^{\infty}$, where

$$
\begin{aligned}
& x_{l}^{\infty}:=\left(I-A^{K_{l}}\right)^{-1}\left(B^{K_{l}}\left(f\left(y_{l}^{\infty}+w^{\infty}\right)-K_{l} y_{l}^{\infty}\right)+\left(B_{\mathrm{e}}+B^{K_{l}} K_{l} D_{\mathrm{e}}\right) v^{\infty}\right), \\
& y_{l}^{\infty}=C^{K_{l}} x^{\infty}+D^{K_{l}}\left(f\left(y_{l}^{\infty}+w^{\infty}\right)-K_{l} y_{l}^{\infty}\right)+\left(I-D K_{l}\right)^{-1} D_{\mathrm{e}} v^{\infty},
\end{aligned}
$$

for $l \in\{1,2\}$.

(b) Assumption (3.3) with $r=\gamma$ and $S_{1}=S_{2}=Y$ may be rewritten as

$$
\sup _{\substack{\zeta, \xi \in Y \\ \zeta \neq 0}} \frac{\|f(\zeta+\xi)-f(\xi)-K \zeta\|_{U}}{\|\zeta\|_{Y}}<\gamma
$$

which trivially implies (4.2) with $S=Y$, and is itself equivalent to the function $\xi \mapsto f(\xi)-K \xi$ being globally Lipschitz with Lipschitz constant smaller than $\gamma$. In this case, arguments similar to those used in the proof of Lemma 4.1 show that the map $I-D^{K}(f-K)$ is bijective and hence, by using Lemma 2.2, for all $x^{0} \in X, v \in V^{\mathbb{Z}_{+}}$and all $w \in Y^{\mathbb{Z}_{+}}$, the initial-value problem (2.5) has a unique solution.

(c) Under the assumptions of Theorem 4.3, a consequence of Lemma 4.1 is that the "steady state gain maps" $\left(v^{\infty}, w^{\infty}\right) \mapsto x^{\infty}$ and $\left(v^{\infty}, w^{\infty}\right) \mapsto y^{\infty}$ are globally Lipschitz.

(d) We recall that a subset $\mathcal{V} \subseteq V^{\mathbb{Z}_{+}} \times Y^{\mathbb{Z}_{+}}$is said to be equi-convergent to $\left(v^{\infty}, w^{\infty}\right) \in V \times Y$ if, for all $\varepsilon>0$, there exists $\tau \in \mathbb{Z}_{+}$such that, for all $(v, w) \in \mathcal{V}$

$$
\left\|\left(\begin{array}{c}
\left(\Lambda_{\tau} v\right)(t) \\
\left(\Lambda_{\tau} w\right)(t)
\end{array}\right)-\left(\begin{array}{c}
v^{\infty} \\
w^{\infty}
\end{array}\right)\right\|_{V \times Y} \leq \varepsilon, \quad \forall t \in \mathbb{Z}_{+} .
$$

The convergence property provided by Theorem 4.3 is uniform in the following sense: given a set of inputs $\mathcal{V} \subseteq V^{\mathbb{Z}_{+}} \times Y^{\mathbb{Z}_{+}}$which is equi-convergent to $\left(v^{\infty}, w^{\infty}\right)$ and $\kappa>0$, the set of solutions

$$
\left\{\left(\begin{array}{l}
x \\
y
\end{array}\right) \in X^{\mathbb{Z}_{+}} \times Y^{\mathbb{Z}_{+}}: \exists\left(\begin{array}{c}
v \\
w
\end{array}\right) \in \mathcal{V} \text { s.t. }(v, w, x, y) \in \mathcal{B} \text { and }\|x(0)\|_{X}+\max _{t \in \mathbb{Z}_{+}}\left\|\left(\begin{array}{c}
v(t) \\
w(t)
\end{array}\right)\right\|_{V \times Y} \leq \kappa\right\},
$$

is equi-convergent to $\left(x^{\infty}, y^{\infty}\right)$. 
Proof of Theorem 4.3. First, statement (i) of Lemma 4.1 yields that $\# \mathcal{T}_{K}=1$. Using Lemma 4.2 gives that $\left(v^{\infty}, w^{\infty}, x^{\infty}, y^{\infty}\right)$ is an equilibrium quadruple of (2.3) and since $y^{\infty}+w^{\infty} \in S$, we have that $\left(v^{\infty}, w^{\infty}, x^{\infty}, y^{\infty}\right) \in \mathcal{B}^{S}$. We invoke statement (ii) of Theorem 3.2, with $q=\infty$, to obtain $c, d>0$ and $a \in(0,1)$ such that for all $(v, w, x, y) \in \mathcal{B}$ and all $t \in \mathbb{Z}_{+}$, we have

$$
\left\|x(t)-x^{\infty}\right\|_{X} \leq c a^{t}\left\|x(0)-x^{\infty}\right\|_{X}+c \max _{s \in \underline{t}}\left\|\left(\begin{array}{c}
v(s) \\
w(s)
\end{array}\right)-\left(\begin{array}{c}
v^{\infty} \\
w^{\infty}
\end{array}\right)\right\|_{V \times Y},
$$

and

$$
\left\|y(t)-y^{\infty}\right\|_{Y} \leq d a^{t}\left\|x(0)-x^{\infty}\right\|_{X}+d \max _{s \in \underline{t}}\left\|\left(\begin{array}{c}
v(s) \\
w(s)
\end{array}\right)-\left(\begin{array}{c}
v^{\infty} \\
w^{\infty}
\end{array}\right)\right\|_{V \times Y} .
$$

Let $(v, w, x, y) \in \mathcal{B}$ and fix $t \in \mathbb{Z}_{+}$. Note that (4.8) and (4.9) hold for

$$
\left(\Lambda_{\lfloor t / 2\rfloor} v, \Lambda_{\lfloor t / 2\rfloor} w, \Lambda_{\lfloor t / 2\rfloor} x, \Lambda_{\lfloor t / 2\rfloor} y\right) \in \mathcal{B},
$$

from the time-invariance property (2.4). In light of the identity $\lceil t / 2\rceil+\lfloor t / 2\rfloor=t$, it follows that

$$
\begin{aligned}
\left\|x(t)-x^{\infty}\right\|_{X} & =\left\|\left(\Lambda_{\lfloor t / 2\rfloor} x\right)(\lceil t / 2\rceil)-x^{\infty}\right\|_{X} \\
& \leq c a^{\lceil t / 2\rceil}\left\|x(\lfloor t / 2\rfloor)-x^{\infty}\right\|_{X}+c \max _{s \in \underline{\lceil t / 2\rceil}}\left\|\left(\begin{array}{c}
\Lambda_{\lfloor t / 2\rfloor} v(s) \\
\Lambda_{\lfloor t / 2\rfloor} w(s)
\end{array}\right)-\left(\begin{array}{c}
v^{\infty} \\
w^{\infty}
\end{array}\right)\right\|_{V \times Y} .
\end{aligned}
$$

Appealing to (4.8) again yields

$$
\begin{aligned}
\left\|x(t)-x^{\infty}\right\|_{X} \leq & c^{2} a^{\lceil t / 2\rceil} a^{\lfloor t / 2\rfloor}\left\|x(0)-x^{\infty}\right\|_{X}+c^{2} a^{\lceil t / 2\rceil} \max _{s \in \underline{\lfloor t / 2\rfloor} \|}\left\|\left(\begin{array}{c}
v(s) \\
w(s)
\end{array}\right)-\left(\begin{array}{c}
v^{\infty} \\
w^{\infty}
\end{array}\right)\right\|_{V \times Y} \\
& +c \max _{s \in \underline{\lceil t / 2\rceil}\rceil}\left\|\left(\begin{array}{c}
\Lambda_{\lfloor t / 2\rfloor} v(s) \\
\Lambda_{\lfloor t / 2\rfloor} w(s)
\end{array}\right)-\left(\begin{array}{c}
v^{\infty} \\
w^{\infty}
\end{array}\right)\right\|_{V \times Y} .
\end{aligned}
$$

Finally, using the properties of the ceiling and floor functions we arrive at

$$
\begin{aligned}
\left\|x(t)-x^{\infty}\right\|_{X} \leq c^{2} a^{t}\left\|x(0)-x^{\infty}\right\|_{X}+c^{2} a^{\lceil t / 2\rceil} \max _{s \in \underline{\lfloor t / 2\rfloor}}\left\|\left(\begin{array}{c}
v(s) \\
w(s)
\end{array}\right)-\left(\begin{array}{c}
v^{\infty} \\
w^{\infty}
\end{array}\right)\right\|_{V \times Y} \\
+c \max _{s \in \underline{\lceil t / 2\rceil}}\left\|\left(\begin{array}{c}
\Lambda_{\lfloor t / 2\rfloor} v(s) \\
\Lambda_{\lfloor t / 2\rfloor} w(s)
\end{array}\right)-\left(\begin{array}{c}
v^{\infty} \\
w^{\infty}
\end{array}\right)\right\|_{V \times Y} .
\end{aligned}
$$

Starting instead from (4.9) and proceeding in the same manner, we obtain a similar estimate for $\left\|y(t)-y^{\infty}\right\|$. Thus, we obtain (4.6) after estimating and relabelling the constants appropriately.

Remark 4.5. By inspecting the above proof we see that in the situation where $\gamma=\infty$ and (3.20) holds with $S_{1}=Y$ and $S_{2}=S$, then the conclusions of Theorem 4.3 remain valid (see Remark 3.4(b)).

As a corollary to Theorem 4.3 (with $S=Y$ ), we see that condition (4.7) is sufficient for the CICS property.

Corollary 4.6. Let $\Sigma \in \mathbb{L}$ be stabilisable and detectable, $K \in \mathbb{S}(\mathbf{G})$ and $\gamma:=1 /\left\|\mathbf{G}^{K}\right\|_{H^{\infty}}$ (where $\gamma:=\infty$ if $\left\|\mathbf{G}^{K}\right\|_{H^{\infty}}=0$ ). If either:

(i) $\gamma<\infty$ and there exists $\delta \in(0, \gamma)$ such that (3.3) holds with $r=\gamma$ and $S_{1}=S_{2}=Y$, or;

(ii) $\gamma=\infty$ and (3.20) holds with $S_{1}=S_{2}=Y$,

then the Lur'e system (2.3) has the CICS property. 
As previously mentioned at the start of this section, if $w$ in (2.3) is considered to be an output disturbance, then it may be unreasonable to expect convergence of $w$. The next result is an immediate corollary to Theorem 4.3 and yields that asymptotic 'closeness' of the state and output of (2.3) to the equilibrium components $x^{\infty}$ and $y^{\infty}$, respectively, is linearly bounded by $\|w\|_{\ell^{\infty}}$.

Corollary 4.7. Under the assumptions of Theorem 4.3 with $w^{\infty}=0$, for all $(v, w, x, y) \in \mathcal{B}$ with $\lim _{t \rightarrow \infty} v(t)=v^{\infty}$

$$
\limsup _{t \rightarrow \infty}\left(\left\|x(t)-x^{\infty}\right\|_{X}+\left\|y(t)-y^{\infty}\right\|_{Y}\right) \leq c \limsup _{t \rightarrow \infty}\|w(t)\|,
$$

where $c>0$ is as in (4.6).

Proof. The claim follows from (4.6), the time-invariance property (2.4), and a standard timeinvariance argument.

\subsection{Periodic inputs}

For given $\tau \in \mathbb{N}$ and normed space $W$, we say that $v \in W^{\mathbb{Z}_{+}}$is $\tau$-periodic if $\Lambda_{\tau} v=v$. We say that $(v, w, x, y) \in \mathcal{B}$ is $\tau$-periodic if each of the functions $v, w, x$ and $y$ is $\tau$-periodic.

Theorem 4.8. Let $\tau \in \mathbb{N}$ and let $v^{\mathrm{p}} \in V^{\mathbb{Z}_{+}}$and $w^{\mathrm{p}} \in Y^{\mathbb{Z}_{+}}$be $\tau$-periodic. If the assumptions of Theorem 3.2 hold with $S_{1}=S_{2}=Y$, then there exist a unique $\tau$-periodic trajectory $\left(v^{\mathrm{p}}, w^{\mathrm{p}}, x^{\mathrm{p}}, y^{\mathrm{p}}\right) \in \mathcal{B}$ and $\kappa>1$ such that

$$
\lim _{t \rightarrow \infty}\left\|\left(x-x^{\mathrm{p}}\right)(t) \kappa^{t}\right\|_{X}=0=\lim _{t \rightarrow \infty}\left\|\left(y-y^{\mathrm{p}}\right)(t) \kappa^{t}\right\|_{Y}, \quad \forall\left(v^{\mathrm{p}}, w^{\mathrm{p}}, x, y\right) \in \mathcal{B} .
$$

Proof. The proof is in part inspired by that of [1, Proposition 4.4]. The hypotheses of Theorem 3.2 hold and so by statement (ii) of that result with $q=\infty$, it follows that there exist $c>0$ and $\theta \in(0,1)$ such that (3.6) holds for all $\left(v_{1}, w_{1}, x_{1}, y_{1}\right),\left(v_{2}, w_{2}, x_{2}, y_{2}\right) \in \mathcal{B}$. An application of statement (ii) of Lemma 4.1 gives that $F_{K}$ is bijective and so, see Remark 4.4 (b), for each $v^{\mathrm{e}} \in V$ and $w^{\mathrm{e}} \in Y$, there exist (unique) $x^{\mathrm{e}} \in X$ and $y^{\mathrm{e}} \in Y$ such that $\left(v^{\mathrm{e}}, w^{\mathrm{e}}, x^{\mathrm{e}}, y^{\mathrm{e}}\right)$ is an equilibrium quadruple of the Lur'e system (2.3). Let $\left(v^{\mathrm{p}}, w^{\mathrm{p}}, x, y\right) \in \mathcal{B}$. Invoking (3.6) with $\left(v^{\mathrm{p}}, w^{\mathrm{p}}, x, y\right)$ and $\left(v^{\mathrm{e}}, w^{\mathrm{e}}, x^{\mathrm{e}}, y^{\mathrm{e}}\right)$, we see that there exists $\mu>0$ such that

$$
\|x(t)\|_{X}+\|y(t)\|_{Y} \leq \mu, \quad \forall t \in \mathbb{Z}_{+},
$$

hence showing that $x$ and $y$ are bounded. Moreover, since $\left(\Lambda_{\sigma} v^{\mathrm{p}}, \Lambda_{\sigma} w^{\mathrm{p}}, \Lambda_{\sigma} x, \Lambda_{\sigma} y\right) \in \mathcal{B}$ for every $\sigma \in \mathbb{Z}_{+}$and $\Lambda_{\sigma} v^{\mathrm{p}}=\Lambda_{\sigma+k \tau} v^{\mathrm{p}}$ and $\Lambda_{\sigma} w^{\mathrm{p}}=\Lambda_{\sigma+k \tau} w^{\mathrm{p}}$ for every $k, \sigma \in \mathbb{Z}_{+}$, statement (ii) of Theorem 3.2 ensures that there exist $c>0$ and $\theta \in(0,1)$ such that

$$
\left\|\left(\Lambda_{\sigma} x-\Lambda_{\sigma+k \tau} x\right)(t)\right\|_{X}+\left\|\left(\Lambda_{\sigma} y-\Lambda_{\sigma+k \tau} y\right)(t)\right\|_{Y} \leq c \theta^{t}\|x(\sigma)-x(\sigma+k \tau)\|_{X}, \quad \forall \sigma, k, t \in \mathbb{Z}_{+} .
$$

Thus, for all $t, n, m \in \mathbb{Z}_{+}$with $m \geq n$, we have

$$
\begin{aligned}
\left\|\left(\Lambda_{n \tau} x-\Lambda_{m \tau} x\right)(t)\right\|_{X}+\left\|\left(\Lambda_{n \tau} y-\Lambda_{m \tau} y\right)(t)\right\|_{Y} & =\left\|\left(\Lambda_{t} x-\Lambda_{t+(m-n) \tau} x\right)(n \tau)\right\|_{X} \\
& \quad+\left\|\left(\Lambda_{t} y-\Lambda_{t+(m-n) \tau} y\right)(n \tau)\right\|_{Y} \\
& \leq 2 \mu c \theta^{n \tau} .
\end{aligned}
$$

Therefore, $\left(\Lambda_{n \tau} x\right)_{n \in \mathbb{Z}_{+}}$and $\left(\Lambda_{n \tau} y\right)_{n \in \mathbb{Z}_{+}}$are Cauchy sequences in $\ell^{\infty}(X)$ and $\ell^{\infty}(Y)$, respectively. We denote their respective limits by $x^{\mathrm{p}}$ and $y^{\mathrm{p}}$. The calculation

$$
x^{\mathrm{p}}(t)=\lim _{n \rightarrow \infty}\left(\Lambda_{n \tau} x\right)(t)=\lim _{n \rightarrow \infty}\left(\Lambda_{(n+1) \tau} x\right)(t)=\lim _{n \rightarrow \infty}\left(\Lambda_{n \tau} x\right)(t+\tau)=x^{\mathrm{p}}(t+\tau), \quad \forall t \in \mathbb{Z}_{+},
$$


shows that $x^{\mathrm{p}}$ is $\tau$-periodic. The $\tau$-periodicity of $y^{\mathrm{p}}$ is proven similarly. We proceed to show that $\left(v^{\mathrm{p}}, w^{\mathrm{p}}, x^{\mathrm{p}}, y^{\mathrm{p}}\right) \in \mathcal{B}$. Indeed, for all $n \in \mathbb{Z}_{+}$, we have that

$$
\begin{aligned}
\left(\Lambda_{n \tau} x\right)^{+} & =A\left(\Lambda_{n \tau} x\right)+B f\left(\Lambda_{n \tau} y+w^{\mathrm{p}}\right)+B_{\mathrm{e}} v^{\mathrm{p}} \\
\left(\Lambda_{n \tau} y\right) & =C\left(\Lambda_{n \tau} x\right)+D f\left(\Lambda_{n \tau} y+w^{\mathrm{p}}\right)+D_{\mathrm{e}} v^{\mathrm{p}} .
\end{aligned}
$$

The estimate (3.3) gives that $f$ is continuous and hence, by taking the limit as $n \rightarrow \infty$ in (4.11) and (4.12), we yield that

$$
\begin{aligned}
\left(x^{\mathrm{p}}\right)^{+} & =A x^{\mathrm{p}}+B f\left(y^{\mathrm{p}}+w^{\mathrm{p}}\right)+B_{\mathrm{e}} v^{\mathrm{p}} \\
y^{\mathrm{p}} & =C x^{\mathrm{p}}+D f\left(y^{\mathrm{p}}+w^{\mathrm{p}}\right)+D_{\mathrm{e}} v^{\mathrm{p}},
\end{aligned}
$$

whence $\left(v^{\mathrm{p}}, w^{\mathrm{p}}, x^{\mathrm{p}}, y^{\mathrm{p}}\right) \in \mathcal{B}$.

Moreover, let $\kappa \in(1,1 / \theta)$ and invoke (3.6) to obtain

$$
\lim _{t \rightarrow \infty}\left(\left(\left\|\left(x-x^{\mathrm{p}}\right)(t)\right\|_{X}+\left\|\left(y-y^{\mathrm{p}}\right)(t)\right\|_{Y}\right) \kappa^{t}\right) \leq \lim _{t \rightarrow \infty} c \kappa^{t} \theta^{t}\left\|\left(x-x^{\mathrm{p}}\right)(0)\right\|_{X}=0 .
$$

Finally, to establish uniqueness of $\left(v^{\mathrm{p}}, w^{\mathrm{p}}, x^{\mathrm{p}}, y^{\mathrm{p}}\right)$, assume that $\left(v^{\mathrm{p}}, w^{\mathrm{p}}, \tilde{x}^{\mathrm{p}}, \tilde{y}^{\mathrm{p}}\right) \in \mathcal{B}$ is also $\tau$ periodic. Then (4.10) implies that $x^{\mathrm{p}}=\tilde{x}^{\mathrm{p}}$ and $y^{\mathrm{p}}=\tilde{y}^{\mathrm{p}}$, hence completing the proof.

\section{Application to four-block Lur'e systems}

In the following, we demonstrate how the results of earlier sections apply to the related class of so-called "four-block" Lur'e systems which are informally described by the block diagram arrangement in Figure 5.1, where $\Sigma=\left(A, B, B_{\mathrm{e}}, C, D, D_{\mathrm{e}}\right) \in \mathbb{L}$ and the signal $y$ is given by

$$
y=\left(\begin{array}{l}
y^{1} \\
y^{2}
\end{array}\right) \text {. }
$$

In this section we use superscripts to denote decompositions of signals, as opposed to subscripts which have been used to distinguish trajectories in the context of incremental stability. The motivation for studying the four-block setting is that there may be outputs which are of interest, but not used for feedback (denoted $y^{1}$ in Figure 5.1).

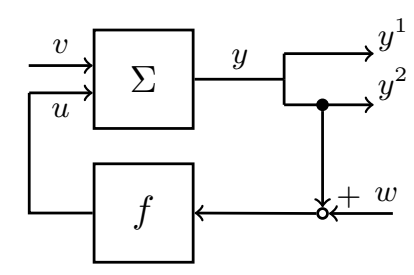

Figure 5.1: Block diagram of a four-block forced Lur'e system: the feedback interconnection of a linear system specified by $\Sigma$ and the static nonlinearity $f$, with output disturbance $w$.

Throughout this section, we assume that the output space $Y$ is of the form $Y=Y^{1} \times Y^{2}$, where $Y^{1}$ and $Y^{2}$ are complex Hilbert spaces, and we define the maps

$$
P^{j}: Y \rightarrow Y^{j},\left(\begin{array}{c}
y^{1} \\
y^{2}
\end{array}\right) \mapsto y^{j}, \quad j=1,2 .
$$

To fix notation, we assume that (only) the component $y^{2}:=P^{2} y$ of $(2.3)$ is used for feedback purposes, giving rise to the Lur'e system

$$
\left.\begin{array}{rl}
x^{+} & =A x+B u+B_{\mathrm{e}} v, \\
y & =C x+D u+D_{\mathrm{e}} v, \\
u & =f\left(y^{2}+w\right),
\end{array}\right\}
$$


where $\Sigma:=\left(A, B, B_{\mathrm{e}}, C, D, D_{\mathrm{e}}\right) \in \mathbb{L}, v \in V^{\mathbb{Z}_{+}}, w \in\left(Y^{2}\right)^{\mathbb{Z}_{+}}$and $f: Y^{2} \rightarrow U$. The forced nonlinear difference equation (5.1) provides the formal description of the feedback system illustrated in Figure 5.1.

The decomposition of the output space $Y=Y^{1} \times Y^{2}$ induces two linear systems, viz.

$$
\left(A, B, B_{\mathrm{e}}, C^{j}, D^{j}, D_{\mathrm{e}}^{j}\right), \quad j=1,2,
$$

where

$$
C^{j}:=P^{j} C, \quad D^{j}:=P^{j} D, \quad D_{\mathrm{e}}^{j}=P^{j} D_{\mathrm{e}} .
$$

In this section, we set $\Sigma^{j}:=\left(A, B, B_{\mathrm{e}}, P^{j} C, P^{j} D, P^{j} D_{\mathrm{e}}\right)$ and we denote the behaviour of (5.1) by $\tilde{\mathcal{B}}$, that is,

$$
\tilde{\mathcal{B}}:=\left\{(v, w, x, y) \in V^{\mathbb{Z}_{+}} \times\left(Y^{2}\right)^{\mathbb{Z}_{+}} \times X^{\mathbb{Z}_{+}} \times Y^{\mathbb{Z}_{+}}:(v, w, x, y) \text { satisfies (5.1) }\right\} .
$$

For $S \subseteq Y^{2}$, we set

$$
\tilde{\mathcal{B}}^{S}:=\left\{(v, w, x, y) \in \tilde{\mathcal{B}}: y^{2}(t)+w(t) \in S, \forall t \in \mathbb{Z}_{+}\right\}
$$

As before, we write $\mathbf{G}(z)=C(z I-A)^{-1} B+D$.

Our main result of this section states that the conclusions of Theorem 3.2 apply to (5.1) provided the linear system $\Sigma^{2}$ and $f$ satisfy the assumptions of Theorem 3.2.

Corollary 5.1. Let $\Sigma \in \mathbb{L}$, let $S_{1}, S_{2} \subseteq Y^{2}$ be non-empty. Assume that $\Sigma^{2}$ is stabilisable and detectable, $r>0$ and $K^{2} \in \mathcal{L}\left(Y^{2}, U\right)$ satisfy $\mathbb{B}\left(K^{2}, r\right) \subseteq \mathbb{S}\left(P^{2} \mathbf{G}\right)$ and that there exists $\delta \in(0, r)$ such that (3.3) holds with $K$ and $Y$ replaced by $K^{2}$ and $Y^{2}$, respectively. Then the conclusions of Theorem 3.2 hold for the Lur'e system (5.1).

Proof. In the following, we shall only prove that statement (i) of Theorem 3.2 holds for (5.1), since the proof of statement (ii) for (5.1) is similar. We shall consider the Lur'e system

$$
\left.\begin{array}{rl}
x^{+} & =A x+B f\left(y^{2}+w\right)+B_{\mathrm{e}} v \\
y^{2} & =C^{2} x+D^{2} f\left(y^{2}+w\right)+D_{\mathrm{e}}^{2} v
\end{array}\right\}
$$

which is obtained from (5.1) by applying $P^{2}$ to the output equation. Note that the Lur'e system (5.2) is of the form (2.3) with $Y, C, D, D_{\mathrm{e}}$ and $y$ replaced by $Y^{2}, C^{2}, D^{2}, D_{\mathrm{e}}^{2}$ and $y^{2}$, respectively.

By hypothesis, the conclusions of Theorem 3.2 apply to (5.2) and so there exist constants $a>0$, $b>0$ and $\omega>1$ such that, for all $\left(v_{1}, w_{1}, x_{1}, y_{1}\right) \in \tilde{\mathcal{B}}^{S_{1}},\left(v_{2}, w_{2}, x_{2}, y_{2}\right) \in \tilde{\mathcal{B}}^{S_{2}}$, and all $\rho \in[1, \omega]$, we have

$$
\left\|\pi_{t}\left(x_{1}-x_{2}\right)\right\|_{\ell_{\rho}^{2}} \leq a\left(\left\|x_{1}(0)-x_{2}(0)\right\|_{X}+\left\|\pi_{t-1}\left(\begin{array}{c}
v_{1}-v_{2} \\
w_{1}-w_{2}
\end{array}\right)\right\|_{\ell_{\rho}^{2}}\right), \quad \forall t \in \mathbb{N},
$$

and

$$
\left\|\pi_{t}\left(y_{1}^{2}-y_{2}^{2}\right)\right\|_{\ell_{\rho}^{2}} \leq b\left(\left\|x_{1}(0)-x_{2}(0)\right\|_{X}+\left\|\pi_{t}\left(\begin{array}{c}
v_{1}-v_{2} \\
w_{1}-w_{2}
\end{array}\right)\right\|_{\ell_{\rho}^{2}}\right), \quad \forall t \in \mathbb{Z}_{+} .
$$

It remains to establish that an estimate of the form (5.4) holds for the difference $y_{1}-y_{2}$. For which purpose, fix $\left(v_{1}, w_{1}, x_{1}, y_{1}\right) \in \tilde{\mathcal{B}}^{S_{1}}$ and $\left(v_{2}, w_{2}, x_{2}, y_{2}\right) \in \tilde{\mathcal{B}}^{S_{2}}$. Since $K^{2} \in \mathbb{A}\left(P^{2} D\right)$, it is easily seen that $K:=K^{2} P^{2} \in \mathbb{A}(D)$. Hence, by using Lemma 2.2 and (5.1), we have that the difference $y_{1}-y_{2}$ satisfies

$$
\begin{aligned}
y_{1}-y_{2}=C^{K}\left(x_{1}-x_{2}\right)+D^{K}\left(f\left(y_{1}^{2}+w_{1}\right)-f\left(y_{2}^{2}+w_{2}\right)\right. & \left.-K^{2}\left(y_{1}^{2}-y_{2}^{2}\right)\right) \\
& +(I-D K)^{-1} D_{\mathrm{e}}\left(v_{1}-v_{2}\right) .
\end{aligned}
$$


Estimating (5.5) by invoking (3.3), (5.3) and (5.4) gives

$$
\begin{aligned}
\left\|\pi_{t}\left(y_{1}-y_{2}\right)\right\|_{\ell_{\rho}^{2}} \leq & \left\|C^{K}\right\|\left\|\pi_{t}\left(x_{1}-x_{2}\right)\right\|_{\ell_{\rho}^{2}}+(r-\delta)\left\|D^{K}\right\|\left\|\pi_{t}\left(y_{1}^{2}-y_{2}^{2}+w_{1}-w_{2}\right)\right\|_{\ell_{\rho}^{2}} \\
& +\left\|(I-D K)^{-1} D_{\mathrm{e}}\right\|\left\|\pi_{t}\left(v_{1}-v_{2}\right)\right\|_{\ell_{\rho}^{2}}+\left\|D^{K} K^{2}\right\|\left\|\pi_{t}\left(w_{1}-w_{2}\right)\right\|_{\ell_{\rho}^{2}} \\
\leq & a\left\|C^{K}\right\|\left(\left\|x_{1}(0)-x_{2}(0)\right\| X+\left\|\pi_{t}\left(\begin{array}{c}
v_{1}-v_{2} \\
w_{1}-w_{2}
\end{array}\right)\right\|_{\ell_{\rho}^{2}}\right) \\
& +b(r-\delta)\left\|D^{K}\right\|\left(\left\|x_{1}(0)-x_{2}(0)\right\|_{X}+\left\|\pi_{t}\left(\begin{array}{c}
v_{1}-v_{2} \\
w_{1}-w_{2}
\end{array}\right)\right\|_{\ell_{\rho}^{2}}\right) \\
& +\zeta\left\|\pi_{t}\left(\begin{array}{c}
v_{1}-v_{2} \\
w_{1}-w_{2}
\end{array}\right)\right\|_{\ell_{\rho}^{2}}
\end{aligned}
$$

for some $\zeta>0$. Thus, setting $\tilde{b}:=a\left\|C^{K}\right\|+b(r-\delta)\left\|D^{K}\right\|+\zeta$, it follows that

$$
\left\|\pi_{t}\left(y_{1}-y_{2}\right)\right\|_{\ell_{\rho}^{2}} \leq \tilde{b}\left(\left\|x_{1}(0)-x_{2}(0)\right\|_{X}+\left\|\pi_{t}\left(\begin{array}{c}
v_{1}-v_{2} \\
w_{1}-w_{2}
\end{array}\right)\right\|_{\ell_{\rho}^{2}}\right), \quad \forall t \in \mathbb{Z}_{+},
$$

completing the proof.

We close the current section by remarking that the various results presented in Sections 3 and 4 for the forced Lur'e system (2.3) also have obvious extensions to the four-block settings considered here, namely system (5.1). For brevity and to avoid repetition, we do not give formal statements of these results.

\section{Application to sampled-data systems}

In this section we provide an application of Theorem 3.2 in the form of an ISS result for a class of forced, infinite-dimensional sampled-data control systems.

Let $A$ be the generator of a strongly continuous semigroup on $X$, denoted by $(\mathbf{T}(t))_{t \geq 0}, B \in$ $\mathcal{L}(U, X)$ and $C \in \mathcal{L}(X, Y)$, and consider the following continuous-time, infinite-dimensional linear system

$$
\left.\begin{array}{l}
\dot{x}=A x+B u+v, \quad x(0)=x^{0} \in X, \\
y=C x .
\end{array}\right\}
$$

As usual, $x$ and $y$ in (6.1) denote the state and output, and $u$ and $v$ are inputs, with the former being available for feedback purposes.

Throughout this section, we assume that

- $X, U$ and $Y$ are Hilbert spaces, with $U$ and $Y$ finite-dimensional;

- the pair $(A, B)$ is (exponentially) stabilisable, that is, there exists $F \in \mathcal{L}(X, U)$ such that the strongly continuous semigroup generated by $A+B F$ is exponentially stable;

- the pair $(C, A)$ is (exponentially) detectable, that is, there exists $H \in \mathcal{L}(Y, X)$ such that the strongly continuous semigroup generated by $A+H C$ is exponentially stable.

Let $\omega(\mathbf{T})$ be the exponential growth constant of $\mathbf{T}$, that is,

$$
\omega(\mathbf{T}):=\lim _{t \rightarrow \infty} \frac{1}{t} \ln \|\mathbf{T}(t)\|
$$


and we let $\mathbf{H}$ denote the transfer function of (6.1), that is, $\mathbf{H}(s)=C(s I-A)^{-1} B$. Furthermore, for $K \in \mathcal{L}(Y, U)$, we define $\mathbf{H}^{K}:=\mathbf{H}(I-K \mathbf{H})^{-1}$. We denote the set of stabilising feedback operators by

$$
\mathbb{S}^{\mathrm{c}}(\mathbf{H}):=\left\{K \in \mathcal{L}(Y, U): \mathbf{H}^{K} \text { is bounded and holomorphic function on } \mathbb{C}_{0}\right\},
$$

where the superscript ' $c$ ' indicates the continuous-time setting.

For a fixed sampling period $\tau>0$, we define the sampling operator $\mathcal{S}: C\left(\mathbb{R}_{+}, Y\right) \rightarrow Y^{\mathbb{Z}_{+}}$as

$$
(\mathcal{S} y)(k):=y(k \tau), \quad \forall y \in C\left(\mathbb{R}_{+}, Y\right), \forall k \in \mathbb{Z}_{+},
$$

and the (zero order) hold operator $\mathcal{H}$ as

$$
(\mathcal{H} u)(t):=u(k), \quad \forall u \in U^{\mathbb{Z}_{+}}, \forall t \in[k \tau,(k+1) \tau),
$$

which maps $U^{\mathbb{Z}_{+}}$into the set of step-functions mapping $[0, \infty)$ to $U$. We shall consider the forced sampled-data Lur'e system arising from the feedback interconnection of (6.1) and the nonlinear sampled-data output feedback control

$$
u=\mathcal{H}(f(\mathcal{S}(y)+w))
$$

where $w \in Y^{\mathbb{Z}_{+}}$is an output disturbance and $f: Y \rightarrow U$ with $f(0)=0$. Thus, for given $x^{0} \in X$, $v \in L_{\text {loc }}^{\infty}\left(\mathbb{R}_{+}, X\right)$ and $w \in Y^{\mathbb{Z}_{+}}$, we consider the initial-value problem

$$
\dot{x}=A x+B \mathcal{H}(f(\mathcal{S}(C x)+w))+v, \quad x(0)=x^{0} \in X,
$$

see Figure 6.1.

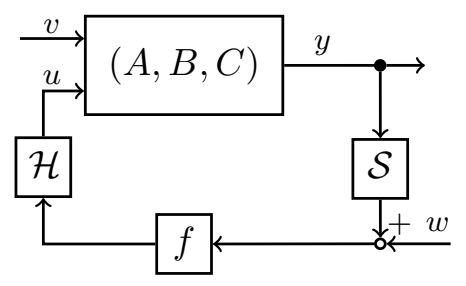

Figure 6.1: Block diagram illustrating the sampled-data Lur'e system (6.3).

We say that $x \in C\left(\mathbb{R}_{+}, X\right)$ is a (mild) solution to (6.3) if $x$ satisfies $x(0)=x^{0}$ and

$$
\left.\begin{array}{rl}
x(k \tau+t)=\mathbf{T}(t) x(k \tau)+\int_{0}^{t} \mathbf{T}( & t-s) B f(C x(k \tau)+w(k)) d s \\
& +\int_{0}^{t} \mathbf{T}(t-s) v(k \tau+s) d s, \quad \forall t \in(0, \tau], \forall k \in \mathbb{Z}_{+} \cdot
\end{array}\right\}
$$

It is clear that, for all $x^{0} \in X, v \in L_{\mathrm{loc}}^{\infty}\left(\mathbb{R}_{+}, X\right)$ and $w \in Y^{\mathbb{Z}_{+}}$, there exists a unique solution of (6.3). Note that if $x^{0}=0, v=0$ and $w=0$, then 0 is a solution of (6.3), as $f(0)=0$.

The sampled-data Lur'e system (6.3) is said to be exponentially input-to-state stable (ISS) if there exist constants $c, \gamma>0$ such that, for all initial states $x^{0} \in X$, all inputs $v \in L_{\text {loc }}^{\infty}\left(\mathbb{R}_{+}, X\right)$ and all output disturbances $w \in Y^{\mathbb{Z}_{+}}$, the solution $x$ of (6.3) satisfies

$$
\|x(k \tau+t)\| \leq c\left(e^{-\gamma(k \tau+t)}\left\|x^{0}\right\|+\|v\|_{L^{\infty}([0, k \tau+t])}+\left\|\pi_{k} w\right\|_{l^{\infty}}\right), \quad \forall t \in(0, \tau], \forall k \in \mathbb{Z}_{+} .
$$

The following theorem gives a sufficient condition for exponential ISS of (6.3). 
Theorem 6.1. Assume that $K \in \mathbb{S}^{c}(\mathbf{H})$ and

$$
\|f(\xi)-K \xi\| \leq r\|\xi\|, \quad \forall \xi \in Y,
$$

where $r<1 / \sup _{s \in \mathbb{C}_{0}}\left\|\mathbf{H}^{K}(s)\right\|$. Then there exists $\tau^{*}>0$ such that (6.3) is exponentially ISS for all $\tau \in\left(0, \tau^{*}\right)$.

We note that under the assumptions of Theorem 6.1, it follows from [16, Theorem 4.1] that the continuous-time Lur'e system

$$
\dot{x}=A x+B f(C x)+v, \quad x(0)=x^{0} \in X,
$$

is exponentially ISS. Theorem 6.1 shows that exponential ISS is inherited by the sample-hold discretization (6.2) of the continuous-time system (6.6), provided the sampling period is sufficiently small.

To facilitate the proof of Theorem 6.1, we state a technical lemma. To this end, for $\tau>0$, we set

$$
A_{\tau}:=\mathbf{T}(\tau), \quad B_{\tau}:=\int_{0}^{\tau} \mathbf{T}(s) B d s \quad \text { and } \quad A_{\tau}^{K}=\left(A_{\tau}\right)^{K}=A_{\tau}+B_{\tau} K C
$$

and, for $L \in \mathcal{L}(Y, U)$ and $r>0$, we let

$$
\mathbb{B}^{\mathrm{cl}}(L, r):=\{M \in \mathcal{L}(Y, U):\|M-L\| \leq r\}
$$

denote the closed ball of radius $r$, centred at $L$.

Lemma 6.2. Let $r>0$ and $K \in \mathcal{L}(Y, U)$ and assume that $\mathbb{B}^{\mathrm{cl}}(K, r) \subseteq \mathbb{S}^{\mathrm{c}}(\mathbf{H})$. Then there exists $\tau^{*}>0$ such that for all $L \in \mathbb{B}^{\mathrm{cl}}(K, r)$ and every $\tau \in\left(0, \tau^{*}\right)$, the operator $A_{\tau}^{L}$ is exponentially stable.

To avoid disruption of the flow of the presentation, the proof of the lemma is placed at the end of this section.

Proof of Theorem 6.1. Let $x^{0} \in X, v \in L_{\mathrm{loc}}^{\infty}\left(\mathbb{R}_{+}, X\right), w \in Y^{\mathbb{Z}_{+}}$and let $x$ be a solution of (6.3). Then, for every $k \in \mathbb{Z}_{+}$and all $t \in(0, \tau], x$ satisfies (6.4). Letting $t=\tau$ in (6.4) and changing variables, it follows that, for every $k \in \mathbb{Z}_{+}$,

$$
x((k+1) \tau)=\mathbf{T}(\tau) x(k \tau)+\int_{0}^{\tau} \mathbf{T}(s) B d s f(C x(k \tau)+w(k))+\int_{0}^{\tau} \mathbf{T}(s) v((k+1) \tau-s) d s .
$$

Setting $x_{k}:=x(k \tau), w_{k}:=w(k)$ and $v_{k}:=\int_{0}^{\tau} \mathbf{T}(s) v((k+1) \tau-s) d s$ for all $k \in \mathbb{Z}_{+}$, we see that $\left(v_{k}, w_{k}, x_{k}\right)$ satisfies the following discrete-time system

$$
\begin{aligned}
x_{k+1} & =A_{\tau} x_{k}+B_{\tau} f\left(C x_{k}+w_{k}\right)+v_{k}, \quad x(0)=x^{0} \in X, \\
y_{k} & =C x_{k} .
\end{aligned}
$$

Let $\rho \in \mathbb{R}$ be such that $r<\rho<1 / \sup _{s \in \mathbb{C}_{0}}\left\|\mathbf{H}^{K}(s)\right\|$. Then, by $\left[15\right.$, Proposition 5.6], $\mathbb{B}^{\text {cl }}(K, \rho) \subseteq$ $\mathbb{S}^{\mathrm{c}}(\mathbf{H})$. Consequently, by Lemma 6.2, there exists $\tau^{*}>0$ such that for all $L \in \mathbb{B}^{\mathrm{cl}}(K, \rho)$ and every $\tau \in\left(0, \tau^{*}\right)$, the operator $A_{\tau}^{L}$ is exponentially stable. Therefore, statement (ii) of Theorem 3.2 yields the existence of constants $c_{1}>0$ and $\theta \in(0,1)$ such that, for all $k \in \mathbb{N}$,

$$
\left\|x_{k}\right\|_{X} \leq c_{1}\left(\theta^{k}\left\|x^{0}\right\|_{X}+\max _{\sigma \in \underline{k-1}}\left\|\left(\begin{array}{c}
v_{\sigma} \\
w_{\sigma}
\end{array}\right)\right\|_{X \times Y}\right) .
$$

Let

$$
\mu:=\tau \sup _{s \in[0, \tau]}\|\mathbf{T}(s)\|
$$


and note that, for all $k \in \mathbb{Z}_{+}$,

$$
\left\|v_{k}\right\|_{X} \leq \mu\|v\|_{L^{\infty}([k \tau,(k+1) \tau])} .
$$

Hence, there exists $c_{2}>0$ such that

$$
\left\|x_{k}\right\|_{X} \leq c_{2}\left(\theta^{k}\left\|x^{0}\right\|_{X}+\mu\|v\|_{L^{\infty}([0, k \tau])}+\left\|\pi_{k-1} w\right\|_{l^{\infty}}\right), \quad \forall k \in \mathbb{Z}_{+} .
$$

It remains to use the discrete-time estimate (6.8) to bound the state $x$ over all times. To this end, note that for all $k \in \mathbb{Z}_{+}$and all $t \in(0, \tau]$,

$$
x(k \tau+t)=\mathbf{T}(t) x_{k}+\int_{0}^{t} \mathbf{T}(s) B d s f(C x(k \tau)+w(k))+\int_{0}^{t} \mathbf{T}(t-s) v(k \tau+s) d s .
$$

Appealing to (6.5), we estimate

$$
\left\|\int_{0}^{t} \mathbf{T}(s) d s B f(C x(k \tau)+w(k))\right\| \leq \mu\|B\|(r+\|K\|)\left(\|C\|\left\|x_{k}\right\|+\left\|w_{k}\right\|\right), \quad \forall t \in(0, \tau] .
$$

Moreover,

$$
\left\|\int_{0}^{t} \mathbf{T}(t-s) B v(k \tau+s) d s\right\| \leq \mu\|v\|_{L^{\infty}([k \tau, k \tau+t])}, \quad \forall k \in \mathbb{Z}_{+}, \forall t \in(0, \tau] .
$$

Taking norms in (6.9) and substituting in (6.10) and (6.11) yields that, for all $k \in \mathbb{Z}_{+}$and all $t \in(0, \tau]$

$$
\|x(k \tau+t)\| \leq(\|\mathbf{T}(t)\|+\mu\|B\|(r+\|K\|)\|C\|)\left\|x_{k}\right\|+\mu\|v\|_{L^{\infty}([k \tau, k \tau+t])}+\mu\|B\|(r+\|K\|)\left\|w_{k}\right\| .
$$

The claim now follows in light of the above inequality and (6.8).

Proof of Lemma 6.2. The proof is a refinement of that of [29, Theorem 3.1]. For $F \in \mathcal{L}(Y, U)$, we let $\mathbf{T}_{F}$ denote the strongly continuous semigroup generated by $A+B F C$. By hypothesis, $\mathbb{B}^{\mathrm{cl}}(K, r) \subseteq \mathbb{S}^{\mathrm{c}}(\mathbf{H}),(A, B)$ is stabilisable and $(C, A)$ is detectable, and so, by [7, Theorem 7.32], for each $F \in \mathbb{B}^{\text {cl }}(K, r)$, there exist $\omega_{F}<0$ and $M_{F} \geq 1$ such that $\left\|\mathbf{T}_{F}(t)\right\| \leq M_{F} e^{\omega_{F} t}$ for all $t \geq 0$. We seek to show that there exists $\omega<0$ and $1 \leq M<\infty$ such that

$$
\left\|\mathbf{T}_{F}(t)\right\| \leq M e^{\omega t}, \quad \forall t \geq 0, \forall F \in \mathbb{B}^{\mathrm{cl}}(K, r) .
$$

To this end, note that for each $F \in \mathbb{B}^{\mathrm{cl}}(K, r)$, there exists $\varepsilon_{F}>0$ such that

$$
\omega_{F}+M_{F}\|B(L-F) C\| \leq \frac{\omega_{F}}{2}, \quad \forall L \in \mathbb{B}\left(F, \varepsilon_{F}\right)
$$

and thus, by [36, Theorem 1.1, Chapter 3],

$$
\left\|\mathbf{T}_{L}(t)\right\| \leq M_{F} e^{\left(\omega_{F} / 2\right) t}, \quad \forall t \geq 0, \forall L \in \mathbb{B}\left(F, \varepsilon_{F}\right) .
$$

The balls $\mathbb{B}\left(F, \varepsilon_{F}\right)$ form an open cover of $\mathbb{B}^{\mathrm{cl}}(K, r)$ and, since $U$ and $Y$ are finite dimensional, $\mathbb{B}^{\mathrm{cl}}(K, r)$ is compact. Hence, there exist finitely many $F_{1}, \ldots, F_{n} \in \mathbb{B}^{\mathrm{cl}}(K, r), \varepsilon_{1}, \ldots, \varepsilon_{n} \in(0, \infty)$ and $\omega_{1}, \ldots, \omega_{n} \in(-\infty, 0)$ such that $\mathbb{B}^{\mathrm{cl}}(K, r) \subseteq \cup_{i=1}^{n} \mathbb{B}\left(F_{i}, \varepsilon_{i}\right)$ and

$$
\left\|\mathbf{T}_{L}(t)\right\| \leq M_{F_{i}} e^{\omega_{i} t}, \quad \forall t \geq 0, \forall L \in \mathbb{B}\left(F_{i}, \varepsilon_{i}\right), \forall i \in\{1, \ldots, n\} .
$$

By setting $M:=\max \left\{M_{F_{1}}, \ldots, M_{F_{n}}\right\}$ and $\omega:=\max \left\{\omega_{1}, \ldots, \omega_{n}\right\}$, it follows that (6.12) holds. 
Next, we claim that for all $\varepsilon>0$, there exists $T>0$ such that

$$
\left\|F C\left(I-\mathbf{T}_{F}(t)\right)\right\|<\varepsilon, \quad \forall F \in \mathbb{B}^{\mathrm{cl}}(K, r), \forall t \in[0, T] .
$$

To prove (6.13), we will show that for all $\varepsilon>0$ and all $F \in \mathbb{B}^{\mathrm{cl}}(K, r)$, there exist $r_{F}>0$ and $T_{F}>0$ such that

$$
\left\|L C\left(I-\mathbf{T}_{L}(t)\right)\right\|<\varepsilon, \quad \forall t \leq T_{F}, \quad \forall L \in \mathbb{B}\left(F, r_{F}\right)
$$

and then use another compactness argument. To this end, fix $\varepsilon>0$ and let $F \in \mathbb{B}^{\text {cl }}(K, r)$. Since $U$ and $Y$ are finite dimensional and $C$ is bounded, it follows that $F C \in \mathcal{L}(X, U)$ is a compact operator. Furthermore, as $X$ is a Hilbert space, [36, Corollary 10.6, Chapter 1] yields that $\mathbf{T}_{F}^{*}$ is a strongly continuous semigroup, and thus

$$
\lim _{t \rightarrow 0}\left(I-\mathbf{T}_{F}(t)\right)^{*} x=0 \quad \forall x \in X .
$$

Therefore, we invoke [29, Lemma 2.1] to yield that

$$
\lim _{t \rightarrow 0}\left\|F C\left(I-\mathbf{T}_{F}(t)\right)\right\|=0 .
$$

Choose $r_{F}>0$ such that

$$
\|L-F\|\|C\| M<\frac{\varepsilon}{6}, \quad \forall L \in \mathbb{B}\left(F, r_{F}\right)
$$

and let $\tilde{T}_{F}>0$ be such that

$$
\left\|F C\left(I-\mathbf{T}_{F}(t)\right)\right\|<\frac{\varepsilon}{3} \quad \forall t \in\left[0, \tilde{T}_{F}\right] .
$$

We invoke [36, Corollary 1.3, Chapter 3] to obtain

$$
\left\|\mathbf{T}_{L}(t)-\mathbf{T}_{F}(t)\right\| \leq M e^{\omega t}\left(e^{M\|B\|\|L-F\|\|C\| t}-1\right) \leq M e^{\omega t}\left(e^{r_{F} M\|B\|\|C\| t}-1\right), \quad \forall L \in \mathbb{B}\left(F, r_{F}\right),
$$

whence

$$
\|L C\|\left\|\mathbf{T}_{L}(t)-\mathbf{T}_{F}(t)\right\| \leq\left(\|F\|+r_{F}\right)\|C\| M e^{\omega t}\left(e^{r_{F} M\|B\|\|C\| t}-1\right), \quad \forall L \in \mathbb{B}\left(F, r_{F}\right) .
$$

Let $\hat{T}_{F}>0$ be such that

$$
\|L C\|\left\|\mathbf{T}_{L}(t)-\mathbf{T}_{F}(t)\right\|<\frac{\varepsilon}{6} \quad \forall t \in\left[0, \hat{T}_{F}\right], \quad \forall L \in \mathbb{B}\left(F, r_{F}\right) .
$$

Setting $T_{F}:=\min \left\{\tilde{T}_{F}, \hat{T}_{F}\right\}$, it follows that, for all $t \in\left[0, T_{F}\right]$ and all $L \in \mathbb{B}\left(F, r_{F}\right)$,

$$
\begin{aligned}
\left\|L C\left(I-\mathbf{T}_{L}(t)\right)\right\| & \leq\|L C-F C\|+\left\|L C \mathbf{T}_{L}(t)-F C \mathbf{T}_{F}(t)\right\|+\left\|F C\left(I-\mathbf{T}_{F}(t)\right)\right\| \\
& <\frac{\varepsilon}{6}+\|L C\|\left\|\mathbf{T}_{L}(t)-\mathbf{T}_{F}(t)\right\|+\|L-F\|\|C\|\left\|\mathbf{T}_{F}(t)\right\|+\frac{\varepsilon}{3} \\
& <\varepsilon .
\end{aligned}
$$

Hence, for all $\varepsilon>0$ and for all $F \in \mathbb{B}^{\text {cl }}(K, r)$, there exists $r_{F}>0$ and $T_{F}>0$ such that

$$
\left\|L C\left(I-\mathbf{T}_{L}(t)\right)\right\|<\varepsilon, \quad \forall t \in\left[0, T_{F}\right], \forall L \in \mathbb{B}\left(F, r_{F}\right) .
$$

A compactness argument similar to that establishing (6.12) can now be used to prove that for all $\varepsilon>0$, there exists $T>0$ such that (6.13) holds.

Finally, we seek to use (6.12) and (6.13) to yield the existence of $\tau^{*}>0$ such that $A_{\tau}^{L}$ is discrete-time exponentially stable for all $L \in \mathbb{B}^{\mathrm{cl}}(K, r)$ and every $\tau \in\left(0, \tau^{*}\right)$. To that end, 
fix $L \in \mathbb{B}^{\text {cl }}(K, r)$. The variation-of-parameters formula for perturbed semigroups [36, equation (1.2), page 77] gives, for all $\tau \geq 0$ and all $x \in X$,

$$
\begin{aligned}
A_{\tau} x+B_{\tau} L C x & =\mathbf{T}(\tau) x+\int_{0}^{\tau} \mathbf{T}(s) B d s L C x \\
& =\mathbf{T}(\tau) x+\int_{0}^{\tau} \mathbf{T}(\tau-s) B L C\left(I-\mathbf{T}_{L}(s)\right) x d s+\int_{0}^{\tau} \mathbf{T}(\tau-s) B L C \mathbf{T}_{L}(s) x d s \\
& =\mathbf{T}_{L}(\tau) x+P_{\tau} x
\end{aligned}
$$

where $P_{\tau} x:=\int_{0}^{\tau} \mathbf{T}(\tau-s) B L C\left(I-\mathbf{T}_{L}(s)\right) x d s$ for all $x \in X$.

As in [29, Theorem 3.1], let us introduce a new norm on $X$ given by

$$
|x|:=\sup _{t \geq 0}\left\|\mathbf{T}_{L}(t) x\right\| e^{-\omega t}, \quad \forall x \in X,
$$

where $\omega<0$ is as in (6.12). Note that

$$
\|x\| \leq|x| \leq M\|x\|, \quad \forall x \in X,
$$

where $M \geq 1$ is as in (6.12). For all $x \in X$ and all $t \geq 0$, we have

$$
\left|\mathbf{T}_{L}(t) x\right|=\sup _{s \geq 0}\left\|\mathbf{T}_{L}(s) \mathbf{T}_{L}(t) x\right\| e^{-\omega s}=\sup _{s \geq 0}\left\|\mathbf{T}_{L}(s+t) x\right\| e^{-\omega(s+t)} e^{\omega t} \leq \sup _{s \geq 0}\left\|\mathbf{T}_{L}(s) x\right\| e^{-\omega s} e^{\omega t} .
$$

Therefore,

$$
\left|\mathbf{T}_{L}(t) x\right| \leq e^{\omega t}|x|, \quad \forall t \geq 0, \forall x \in X .
$$

For $G \in \mathcal{L}(X)$, let $|G|$ denote the operator norm of $G$ induced by the new norm, that is,

$$
|G|=\sup _{\substack{x \in X \\ x \neq 0}} \frac{|G x|}{|x|} .
$$

Combining (6.14) with (6.15), (6.16) and the inequality

$$
e^{\omega \tau} \leq 1+\omega \tau e^{\omega \tau}, \quad \forall \tau \in \mathbb{R}_{+},
$$

we obtain that

$$
\begin{aligned}
\left|A_{\tau}+B_{\tau} L C\right| & \leq e^{\omega \tau}+M\left\|P_{\tau}\right\| \\
& \leq 1+\omega \tau+\left(\omega\left(-1+e^{\omega \tau}\right)+h(\tau)\right) \tau, \quad \forall \tau \in \mathbb{R}_{+},
\end{aligned}
$$

where

$$
h(\tau):=M \sup _{s \in[0, \tau]}\left\|\mathbf{T}(\tau-s) B L C\left(I-\mathbf{T}_{L}(s)\right)\right\| .
$$

Combining this with (6.13) shows that, for fixed $\delta \in(0,-\omega)$, there exists $\tau^{*}>0$ (independent of $\left.L \in \mathbb{B}^{\text {cl }}(K, r)\right)$ such that

$$
\left|A_{\tau}+B_{\tau} L C\right|<1+(\omega+\delta) \tau<1, \quad \forall \tau \in\left(0, \tau^{*}\right) .
$$

Finally, invoking (6.15), we obtain that, for all $\tau \in\left(0, \tau^{*}\right)$ and all $n \in \mathbb{Z}_{+}$,

$$
\left\|\left(A_{\tau}+B_{\tau} L C\right)^{n} x\right\| \leq\left|\left(A_{\tau}+B_{\tau} L C\right)^{n} x\right| \leq\left|A_{\tau}+B_{\tau} L C\right|^{n}|x| \leq M\left|A_{\tau}+B_{\tau} L C\right|^{n}\|x\|, \quad \forall x \in X .
$$

In light of (6.17), the above inequality yields the exponential stability of $A_{\tau}^{L}$ for all $L \in \mathbb{B}^{\mathrm{cl}}(K, r)$ and all $\tau \in\left(0, \tau^{*}\right)$, completing the proof. 


\section{$7 \quad$ Examples}

We conclude the paper with a detailed discussion of two examples.

Example 7.1. Consider the following controlled and observed heat equation describing the temperature evolution in a unit rod

$$
\left.\begin{array}{rl}
z_{t}(\xi, t) & =z_{\xi \xi}(\xi, t)+2 \chi_{[1 / 2,1]}(\xi) u(t)+b_{\mathrm{e}}(\xi) v(t), \\
z_{\xi}(0, t) & =z_{\xi}(1, t)=0, z(\xi, 0)=z^{0}, \\
y(t) & =2 \int_{0}^{\frac{1}{2}} z(t, \zeta) d \zeta
\end{array}\right\} \quad \xi \in(0,1), t>0
$$

Here $z(\xi, t)$ denotes the temperature of the rod at position $\xi$ and time $t, z^{0} \in L^{2}(0,1)$ is the initial temperature distribution, $\chi_{[1 / 2,1]}$ is the indicator function of the interval $[1 / 2,1]$ and $b_{\mathrm{e}} \in L^{2}(0,1)$. Further, $u$ and $v$ are inputs and $y$ is the output (or observation). It is shown in [7, Example 4.3.11] that (7.1) (with $v=0$ ) may be written in the form (6.1), with state-space $X=L^{2}(0,1), A$ the Laplacian with zero Neumann boundary conditions, and bounded $B$ and $C$ operators. Furthermore, $(A, B)$ is stabilisable and $(C, A)$ is detectable by [7, Example 5.2.8]. The transfer function $\mathbf{H}$ from $u$ to $y$ is given by

$$
\mathbf{H}(s)=\frac{2 \tanh (\sqrt{s} / 2)}{s \sqrt{s}},
$$

which has a simple pole at $s=0$ and so (7.1) is neither exponentially nor input-output stable. To illustrate the sampled-data control results of Section 6, we consider the following problem: find conditions which are sufficient for the sampled-data system given by (7.1) and the feedback (6.2) to be exponentially ISS.

Writing $\mathbf{L}(s):=s \mathbf{H}(s)$ enables us to exploit the results of [31] to compute stabilising gains for $\mathbf{H}$. For which purpose, we note that $\mathbf{L}(0)=1>0$ and that $\mathbf{L}$ is bounded and holomorphic on $\{s \in \mathbb{C}: \operatorname{Re}(s)>\alpha\}$ for every $\alpha>-\pi^{2}$. Setting

$$
\lambda:=2 \sup _{\omega \in \mathbb{R}}\left|\operatorname{Re} \frac{\mathbf{L}(i \omega)-\mathbf{L}(0)}{i \omega}\right|>0
$$

an application of [31, Lemma 3.1 and Corollary 3.4] yields that

$$
\sup _{s \in \mathbb{C}}\left|\mathbf{H}^{-k}(s)\right|=1 / k, \quad \forall k \in(0,1 / \lambda) .
$$

In light of [15, Proposition 5.6], it follows that

$$
\mathbb{B}(-k, k) \subseteq \mathbb{S}^{\mathrm{c}}(\mathbf{H}), \quad \forall k \in(0,1 / \lambda),
$$

whence, for all $\rho \in(0,1)$,

$$
\mathbb{B}^{\mathrm{cl}}(-k, \rho k) \subseteq \mathbb{B}(-k, k) \subseteq \mathbb{S}^{\mathrm{c}}(\mathbf{H}), \quad \forall k \in(0,1 / \lambda) .
$$

Consequently, Theorem 6.1 ensures that, for all $k \in(0,1 / \lambda)$ and all $\rho \in(0,1)$, if $f: \mathbb{R} \rightarrow \mathbb{R}$ is such that

$$
|f(\theta)+k \theta| \leq \rho k|\theta|, \quad \forall \theta \in \mathbb{R},
$$

then there exists $\tau^{*}>0$ such that the sampled-data feedback interconnection of (7.1) and (6.2) is exponentially ISS for all sampling/hold periods $\tau \in\left(0, \tau^{*}\right)$. 


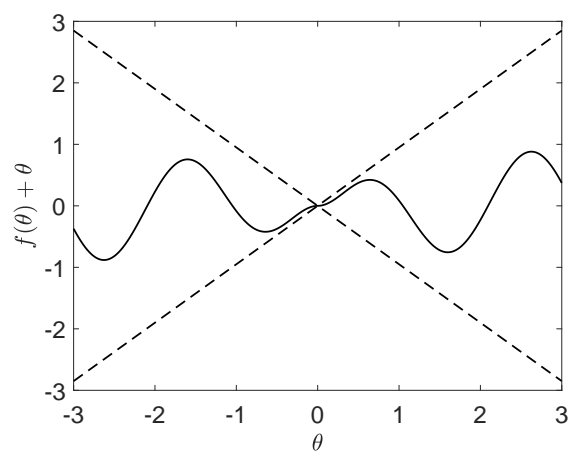

Figure 7.1: Graph of $f(\theta)+\theta$ against $\theta$, for $f$ in (7.4). The dashed lines have gradient \pm 0.95 .

For a numerical illustration, we first compute numerically that $\lambda \approx 0.3707$, so that $5 / 2<1 / \lambda$. We take $k=1, \rho=0.95$ and

$$
f: \mathbb{R} \rightarrow \mathbb{R}, \quad f(\theta)=-\theta+0.95\left(1-\mathrm{e}^{-|\theta|}\right) \sin (3 \theta),
$$

so that (7.2) and (7.3) both hold. Note that $f$ is Lipschitz, but has Lipschitz constant bigger than one. Figure 7.1 illustrates the sector condition (7.3).

We set $b_{\mathrm{e}}=\chi_{[1 / 4,1 / 2]}$, and define the constant and periodic input $v_{1}=3$ and $v_{2}(t)=3 \sin (2 t)$ for all $t \geq 0$, respectively. Certain simulations use the initial temperature distribution $z^{0}(\xi)=$ $e^{-|\xi-1 / 2|^{2}}$. We simulate the closed-loop feedback system (7.1) and (6.2) by performing a semidiscretization in space using a finite-element method with 31 elements, the details of which are given in Appendix B. Figures 7.3(a)-7.3(d) show plots of $\|x(t)\|$ against $t$ in the following situations described in Table 7.2. Simulation data are also listed in Table 7.2.

\begin{tabular}{|c|c|c|c|}
\hline Figure & Initial condition $z(\cdot, 0)$ & Input $v$ & Sampling/hold period $\tau$ \\
\hline $7.3(\mathrm{a})$ & $j^{2} z^{0}$ & 0 & 0.25 \\
$7.3(\mathrm{~b})$ & 0 & $j v_{1}$ & 0.25 \\
$7.3(\mathrm{c})$ & 0 & $j v_{2}$ & 0.25 \\
$7.3(\mathrm{~d})$ & $z^{0}$ & 0 & $1,2,2.5$ \\
\hline
\end{tabular}

Table 7.2: Model parameters used in the numerical simulations in Example 7.1. Here $j \in$ $\{1,2,3\}$.

In Figure 7.3(a), we see the exponential stability property of the unforced $(v=0)$ sampled-data feedback system - a consequence of exponential ISS. In Figures 7.3(a) and 7.3(c), we see the ISS property - the state is bounded in the presence of persistent inputs and, as might be expected, $\|x(t)\|$ increases as $\|v\|_{L^{\infty}(0, t)}$ increases.

To conclude the example, we comment that although taking $\tau=0.25$ appears to "work", in the sense that the numerical results agree with what the theory predicts, in fact the constant $\tau^{*}$, the existence of which is guaranteed by Theorem 6.1, could be either smaller or larger than 0.25. Determining the maximal $\tau^{*}$ analytically or numerically is a difficult open problem. It seems that Figure 7.3(d) shows divergence when $\tau=2.5$, indicating that $\tau^{*}<2.5$.

Example 7.2. We consider a forced Integral Projection Model (IPM) for the monocarpic plant Platte thistle (Cirsium canescens), based on the model presented in [5, 39]. For a recent overview of IPMs we refer the reader to [33]. Platte thistle is a perennial plant native to central North America. The IPM describes the distribution of plant size, according to the natural logarithm 


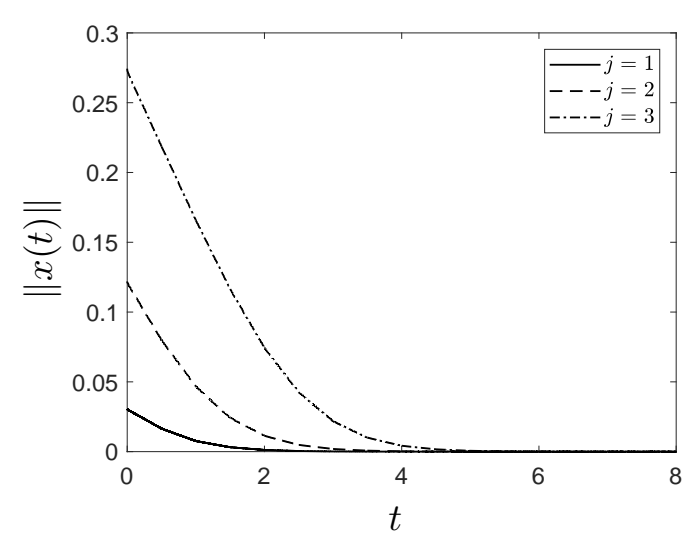

(a)

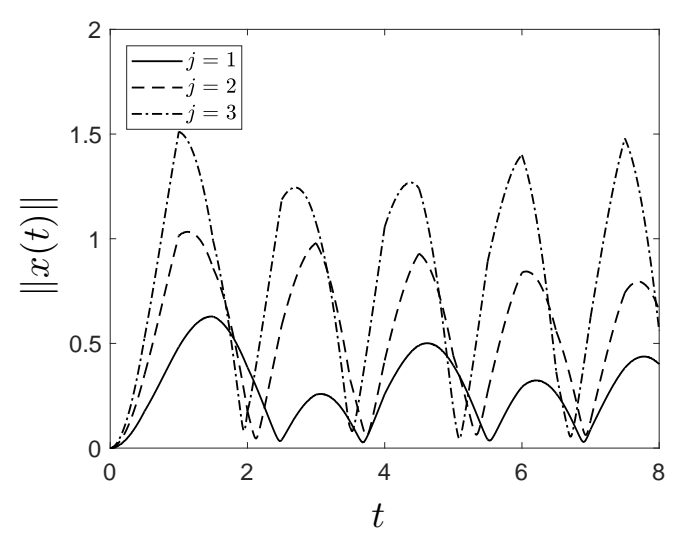

(c)

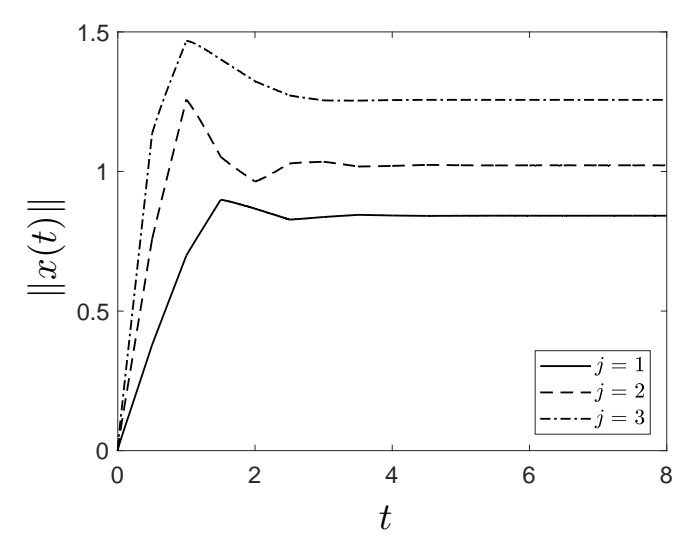

(b)

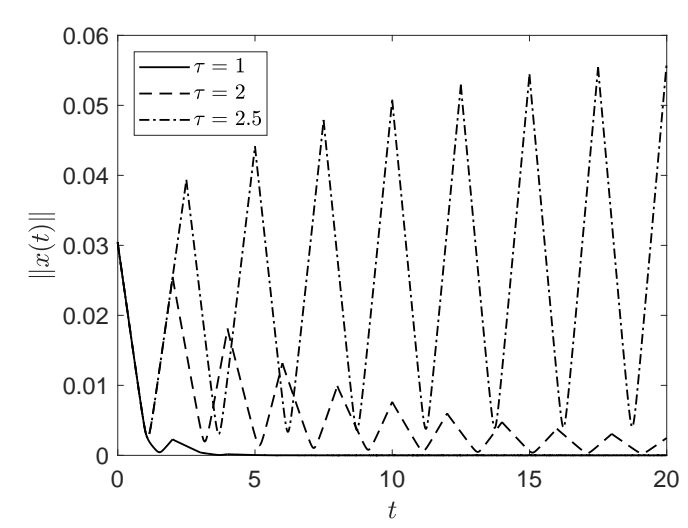

(d)

Figure 7.3: Numerical simulation of the sampled-data feedback interconnection of (7.1) and (6.2) from Example 7.1.

of the crown diameter in mm. Following [5], we assume that the continuous variable of natural logarithm of crown diameter takes minimum and maximum values given by $m_{1}=-0.5$ and $m_{2}=3.5$ (so that roughly $e^{m_{1}}=0.6 \mathrm{~mm}$ and $e^{m_{2}}=33 \mathrm{~mm}$ ), respectively, and that the timesteps correspond to years. Incorporating an additive input, the model is

$$
\left.\begin{array}{rl}
\eta(t+1, \xi)= & \int_{m_{1}}^{m_{2}} p(\xi, \zeta) \eta(t, \zeta) d \zeta+b(\xi) h\left(c^{*} \eta(t, \xi)\right) c^{*} \eta(t, \xi) \\
& +b_{\mathrm{e}}(\xi) v(t), \\
\eta(0, \xi) & \eta_{0}(\xi)
\end{array}\right\} \begin{aligned}
& \forall t \in \mathbb{Z}_{+} \\
& \text {a.e. } \xi \in\left[m_{1}, m_{2}\right],
\end{aligned}
$$

where $\eta(t, \cdot)$ denotes the distribution of plant size at time-step $t$, with initial distribution $\eta_{0} \in L^{1}\left(\left[m_{1}, m_{2}\right]\right)$. In the following, our aim is to write $(7.5)$ in the form of a forced, infinitedimensional Lur'e system (2.3) with the natural state space $X=L^{1}(\Omega)$, where $\Omega:=\left[m_{1}, m_{2}\right]$. Before doing this, we provide some commentary on the model (7.5).

The first term on the right hand side of the difference equation in (7.5) models survival and growth of existing plants. Here $p(\xi, \zeta)$ denotes the probability of an individual of size $\zeta$ surviving to one of size $\xi$ in one time-step, and is assumed in $[5,39]$ to have the structure

$$
p(\xi, \zeta)=s(\zeta)\left(1-f_{\mathrm{p}}(\zeta)\right) g(\xi, \zeta), \quad \forall \xi, \zeta \in \Omega
$$

where $s(\zeta)$ is the survival probability of an individual of size $\zeta, f_{\mathrm{p}}(\zeta)$ is the probability that an individual of size $\zeta$ flowers, and $g(\xi, \zeta)$ is the probability of an individual of size $\zeta$ growing to 
size $\xi$, each over one time-step. We take $s, f_{\mathrm{p}}$ and $g$ as in [5, Table 2]. The term $1-f_{\mathrm{p}}$ appears on the right-hand side of (7.6) as flowering is fatal to Platte thistle, that is, it is monocarpic.

The second term on the right hand side of the difference equation in (7.5) models reproduction and recruitment into the population. In particular, $b \in X$ denotes the distribution of offspring plant size, $c^{*} x$ equals the total number of new seeds recruited into the population by the distribution $z \in X$ in one time-step, and is given by

$$
c^{*} z=\int_{\Omega} s(\theta) f_{\mathrm{p}}(\theta) S(\theta) z(\theta) d \theta, \quad \forall z \in X .
$$

In addition to the terms in (7.6), $S(\theta)$ denotes the number of seeds produced on average by a plant of size $\theta$. We take $b=J$, where $J$ is as in [5, Table 2], and the function $S$ is given in [5, Table 2]. We have $c^{*} \in X^{*}$ as $\theta \mapsto s(\theta) f_{\mathrm{p}}(\theta) S(\theta) \in L^{\infty}(\Omega)$. The function $h$ in (7.5) denotes the probability of seed germination, and is a nonlinear function of the total number of seeds produced, and so seeks to model density-dependence in the seed germination probability. As such, it is assumed to be non-increasing, representing competition or crowding affects at higher seed abundances. Two situations are explored in [5]: first, $h$ is constant with value 0.067, and; second, $h$ is defined by $h(s)=s^{-0.33}$. We note that there is uncertainty in modelling nonlinear terms for Platte thistle, see [11], and in order to demonstrate different settings where the incremental condition (3.3) holds, we shall choose a different $h$ below.

The third term term on the right hand side of the difference equation in (7.5) is an additive input, which may be the arrival of new plants via planned replanting schemes, or accidental movement. We assume that $b_{\mathrm{e}} \in X$ and $v \in\left(\mathbb{R}_{+}\right)^{\mathbb{Z}_{+}}$, which capture the distribution and magnitude, respectively.

We define the integral operator $A: X \rightarrow X$ by

$$
(A x)(\cdot)=\int_{\Omega} p(\cdot, \zeta) x(\zeta) d \zeta, \quad \forall x \in X,
$$

and impose the ecologically reasonable assumption

$$
\sup _{\zeta \in \Omega} \int_{\Omega} p(\xi, \zeta) d \xi<1
$$

which corresponds to some positive level of mortality in the population at all sizes. An application of [26, Theorem 1, p. 173] yields that $\|A\|<1$.

Combining the above, and setting $x(t)=\eta(t, \cdot)$ for all $t \in \mathbb{Z}_{+}$, we see that (7.5) may be written as a forced Lur'e system,

$$
x^{+}=A x+b f\left(c^{*} x\right)+b_{\mathrm{e}} v,
$$

on the state-space $X=L^{1}(\Omega)$, and with $U=V=Y=\mathbb{R}$. Here $f(s):=h(s) s$ for $s \geq 0$ and we extend $h$ and $f$ to all of $\mathbb{R}$ by setting $h(s)=f(s)=0$ for $s \in(-\infty, 0)$. The extension is to ensure that $f$ is defined on the whole of $Y$, so that the results of the paper are applicable.

We seek to apply Theorems 3.2, 4.3 and 4.8 to (7.7) to infer various (incremental) stability and convergence notions. To simulate (7.7) we use a finite-element approximation, the details of which are given in Appendix C.

The property $\|A\|<1$ implies that $\mathbb{B}(0, r) \subseteq \mathbb{S}(\mathbf{G})$ for all $r \in\left(0,1 /\|\mathbf{G}\|_{H^{\infty}}\right)$, where $\mathbf{G}(z)=$ $c^{*}(z I-A)^{-1} b$. Moreover, by [14, Proposition 3.1], we have that $\|\mathbf{G}\|_{H^{\infty}}=\mathbf{G}(1)$, and we compute numerically that $\mathbf{G}(1) \approx 43.8$. We propose a negative sigmoid type function for $h: \mathbb{R} \rightarrow \mathbb{R}_{+}$, namely

$$
h(s)= \begin{cases}0 & s<0, \\ \frac{\rho_{1}}{1+e^{\rho_{2}\left(s-\rho_{3}\right)}} & s \geq 0,\end{cases}
$$


where $\rho_{1}, \rho_{2}$ and $\rho_{3}$ are positive parameters. Broadly, $\rho_{1}$ captures the probability of germination at low abundance, $\rho_{2}$ determines the rate of transition and $\rho_{3}$ the value at which the transition occurs. Figure 7.4 contains plots of $h$ for several parameter values.

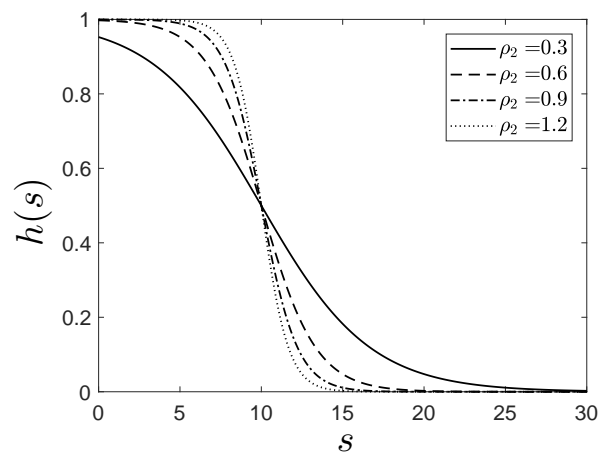

Figure 7.4: Graph of function $h$ in (7.8) for nonnegative arguments. Here $\rho_{1}=1$ and $\rho_{3}=10$, and $\rho_{2}$ varies.

Since $h$ is nonnegative-valued and nonincreasing, it follows that there exists $\delta>0$ such that $s \mapsto f(s)=h(s) s$ satisfies (3.3) with $K=0, S_{1}=\mathbb{R}, S_{2}=\{0\}$ and $r=1 / \mathbf{G}(1)$ whenever

$$
h(0)=\frac{\rho_{1}}{1+\mathrm{e}^{-\rho_{2} \rho_{3}}}<\frac{1}{\mathbf{G}(1)} .
$$

If (7.9) holds, then Theorem 3.2 yields that (7.7) is exponentially ISS. If the inequality

$$
\sup _{s>0}\left|f^{\prime}(s)\right|<\frac{1}{\mathbf{G}(1)},
$$

holds, then $f$ satisfies (3.3) with $K=0, S_{1}=S_{2}=\mathbb{R}$, and hence Theorem 3.2 yields that (7.7) is exponentially $\delta$ ISS. In this case, it also follows from Theorem 4.3 that (7.7) has the CICS property. Moreover, the inequality (7.10) is sufficient for the hypotheses of Theorem 4.8 to hold, which ensures that (7.7) admits a periodic trajectory when subject to periodic inputs, and that all other trajectories generated by the same periodic input asymptotically approach this trajectory.

Numerical simulations are plotted in Figure 7.6. Throughout we take

$$
\rho_{1}=\frac{0.9}{\mathbf{G}(1)}, \quad \rho_{2}=2, \quad \rho_{3}=20,
$$

and with these parameter values it can be shown that $f$ satisfies (7.10). Additional simulation data are recorded in Table 7.5. Panels (a)-(d) respectively show: the 0-GES property; ISS; incremental stability; and asymptotically periodic response to periodic forcing. Note that panel (d) shows that, asymptotically, the responses to the same input are identical and do not depend on the initial conditions, thereby illustrating a typical aspect of ISS. 


\begin{tabular}{|c|c|c|}
\hline Figure & Initial condition $x(0)$ & Input $v$ \\
\hline $7.6(\mathrm{a})$ & Random with $\|x(0)\|=4 j^{2}$ & 0 \\
$7.6(\mathrm{~b})$ & 0 & $v(t)$ small random perturbation of $4 j^{2}$ \\
$7.6(\mathrm{c})$ & Random with $\|x(0)\|=4 j^{2}$ & $v^{1}(t)$ and $v^{2}(t)$ convergent to $4 j^{2}$ \\
$7.6(\mathrm{~d})$ & Random with $\|x(0)\|=4 j^{2}$ & $v(t)=4 \sin (2 \pi t / 12)$ \\
\hline
\end{tabular}

Table 7.5: Model parameters used in the numerical simulations in Example 7.2, where $j \in$ $\{1,2,3\}$.

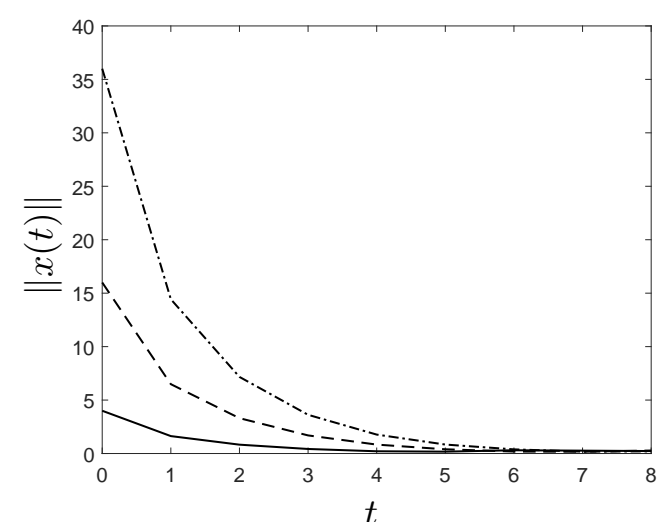

(a)

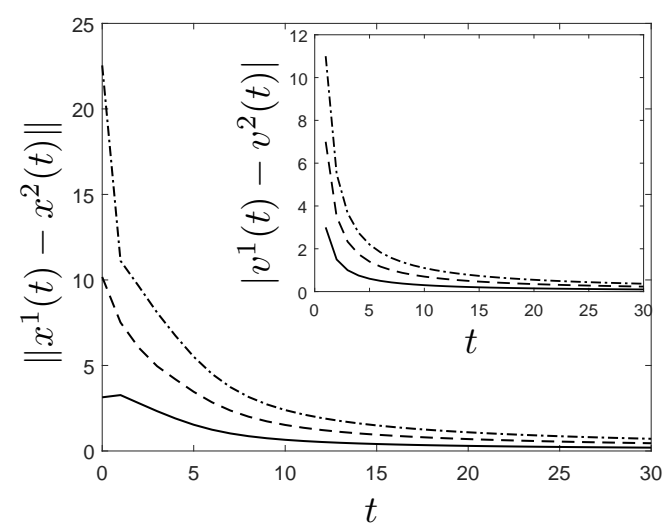

(c)

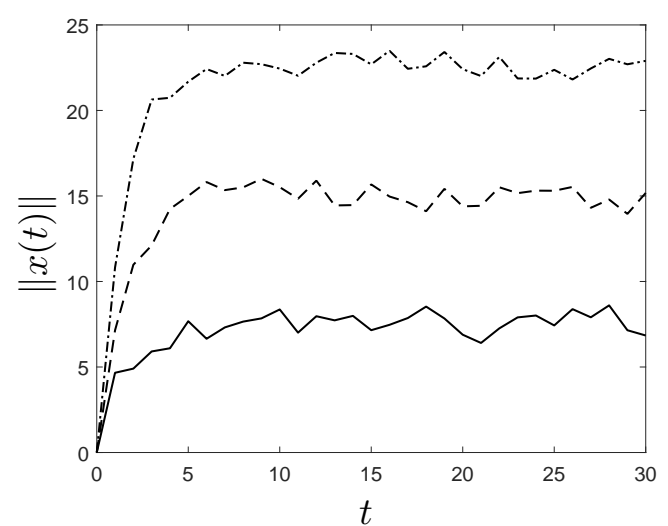

(b)

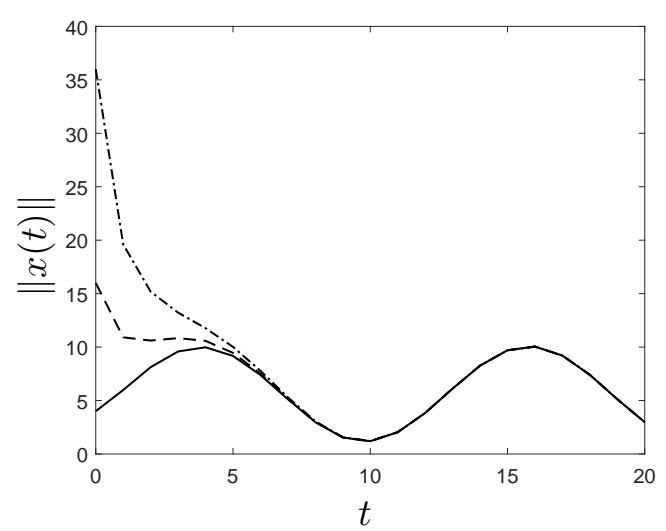

(d)

Figure 7.6: Numerical simulation of the IPM (7.5) from Example 7.2. In each panel the solid, dashed and dashed-dotted lines correspond to $j=1,2,3$, respectively.

\section{Appendices}

\section{A Proofs of technical lemmas}

We give the proofs of several technical lemmas not provided in the main text.

Proof of Lemma 2.2. Let $(u, v, x, y) \in \mathcal{B}^{\operatorname{lin}}$. Then $(I-D L) y=C x+D(u-L y)+D_{\mathrm{e}} v$ and so,

$$
y=C^{L} x+D^{L}(u-L y)+(I-D L)^{-1} D_{\mathrm{e}} v .
$$


Furthermore, noting that $B^{L}=B(I-L D)^{-1}=B+B L D^{L}$ and invoking (A.1),

$$
\begin{aligned}
x^{+} & =A^{L} x+B u+B_{\mathrm{e}} v-B L(I-D L)^{-1} C x \\
& =A^{L} x+B u+B_{\mathrm{e}} v-B L y+B L D^{L}(u-L y)+B L(I-D L)^{-1} D_{\mathrm{e}} v \\
& =A^{L} x+\left(B+B L D^{L}\right)(u-L y)+B_{\mathrm{e}} v+B^{L} L D_{\mathrm{e}} v \\
& =A^{L} x+B^{L}(u-L y)+\left(B_{\mathrm{e}}+B^{L} L D_{\mathrm{e}}\right) v,
\end{aligned}
$$

as required. The converse can be proven by reversing the previous argument and therefore we omit the proof.

Proof of Lemma 3.5. We begin by showing that $\Sigma_{\mathbf{H}} \cap \mathbb{E}=\emptyset$. Seeking a contradiction, suppose that $\Sigma_{\mathbf{H}} \cap \mathbb{E} \neq \emptyset$ and let $z_{0} \in \Sigma_{\mathbf{H}} \cap \mathbb{E}$. Then, there exists a punctured open disc $\Delta:=\{z \in \mathbb{C}$ : $\left.\left|z-z_{0}\right|<\varepsilon, z \neq z_{0}\right\}$ centred at $z_{0}$ and of radius $\varepsilon>0$ such that

$$
\mathbf{H}(z)=\sum_{-\infty}^{\infty} H_{j}\left(z-z_{0}\right)^{j}, \quad \forall z \in \Delta,
$$

where $H_{j} \in \mathcal{L}(U)$ for all $j \in \mathbb{Z}$. For $u \in U$, define

$$
J_{u}:=\left\{j>0:\left\langle H_{-j} u, u\right\rangle \neq 0\right\} .
$$

Let $v \in U$ be such that $J_{v} \neq \emptyset$. Such a $v \in U$ does exist, because otherwise Lemma 2.1 in [15] would yield that $H_{-j}=0$ for every $j>1$ and so $z_{0}$ would not be a singularity, thus yielding a contradiction. Define $h \in H^{*}(\mathbb{C})$ by $h(z)=\langle\mathbf{H}(z) v, v\rangle$ for all $z \in \mathbb{E}$. If $J_{v}$ is infinite, then $h$ has an essential singularity at $z_{0}$ and so, using the Casorati-Weierstrass theorem ([41, Theorem 10.21]), there exists $z^{*} \in \Delta$ such that

$$
\operatorname{Re}\left\langle\mathbf{H}\left(z^{*}\right) u, u\right\rangle=\operatorname{Re} h\left(z^{*}\right)<0,
$$

contradicting the positive realness of $\mathbf{H}$.

Now, assume that $J_{v}$ is finite and set $k:=\max J_{v}$. In this case, $h$ has a pole of order $k$ at $z_{0}$ and so $h$ can be written as

$$
h(z)=\frac{h_{0}+g(z)}{\left(z-z_{0}\right)^{k}}, \quad \forall z \in \Delta,
$$

where $h_{0} \neq 0, g$ is holomorphic on $\Delta \cup\left\{z_{0}\right\}$ and $g\left(z_{0}\right)=0$. For sufficiently small $r>0$, we have

$$
h\left(z_{0}+r e^{i \theta}\right)=r^{-k} e^{-i k \theta}\left(h_{0}+g\left(z_{0}+r e^{i \theta}\right)\right), \quad \forall \theta \in(-\pi, \pi] .
$$

Let $\theta_{0} \in(-\pi, \pi]$ be such that $\operatorname{Re}\left(e^{-i k \theta_{0}} h_{0}\right)<0$. Note that, using that $g\left(z_{0}\right)=0$, we obtain, for sufficiently small $r>0$,

$$
\left\langle\operatorname{Re} \mathbf{H}\left(z_{0}+r e^{i \theta_{0}}\right) v, v\right\rangle=\operatorname{Re} h\left(z_{0}+r e^{i \theta_{0}}\right)<0,
$$

Contradicting the positive realness of $\mathbf{H}$. Consequently $\mathbf{H}$ does not have any singularities in $\mathbb{E}$. Finally, we show that $\mathbf{H}$ is holomorphic at infinity. We define $\tilde{\mathbf{H}}: \mathbb{D} \backslash\{0\} \rightarrow \mathcal{L}(U, Y)$ by

$$
\tilde{\mathbf{H}}(z):=\mathbf{H}(1 / z), \quad \forall z \in \mathbb{D} \backslash\{0\}
$$

and note that $\operatorname{Re}\langle\tilde{\mathbf{H}}(z) u, u\rangle \geq 0$ for all $u \in U$ and $z \in \mathbb{D}, z \neq 0$. Thus, by supposing that $0 \in \Sigma_{\tilde{\mathbf{H}}}$ and by following the same method as above, we obtain a contradiction, completing the proof. 
Proof of Lemma 4.1. (i) Let $\xi \in Y$ be such that $F_{K}^{-1}(\xi) \cap S \neq \emptyset$. Let $\xi_{1} \in F_{K}^{-1}(\xi) \cap S$ and $\xi_{2} \in F_{K}^{-1}(\xi)$. We seek to show $\xi_{1}=\xi_{2}$. Note that

$$
\begin{aligned}
\left\|\xi_{2}-\xi_{1}\right\|_{Y} & =\left\|F_{K}\left(\xi_{2}\right)+\mathbf{G}^{K}(1)\left(f\left(\xi_{2}\right)-K \xi_{2}\right)-F_{K}\left(\xi_{1}\right)-\mathbf{G}^{K}(1)\left(f\left(\xi_{1}\right)-K \xi_{1}\right)\right\|_{Y} \\
& \leq\left\|\mathbf{G}^{K}\right\|_{H^{\infty}}\left\|f\left(\xi_{2}\right)-f\left(\xi_{1}\right)-K\left(\xi_{2}-\xi_{1}\right)\right\|_{U} .
\end{aligned}
$$

Seeking a contradiction, suppose that $\xi_{1} \neq \xi_{2}$. Then, by (4.2), we obtain that

$$
\left\|\xi_{2}-\xi_{1}\right\|_{Y}<\gamma\left\|\mathbf{G}^{K}\right\|_{H^{\infty}}\left\|\xi_{2}-\xi_{1}\right\|_{Y}=\left\|\xi_{2}-\xi_{1}\right\|_{Y},
$$

which is a contradiction. Hence, $\xi_{1}=\xi_{2}$ and $\# F_{K}^{-1}(\xi)=1$.

(ii) By (4.7), there exists $\lambda<\gamma$ such that $\|f(\zeta+\xi)-f(\xi)-K \zeta\|_{U} \leq \lambda\|\zeta\|_{Y}$ for all $\zeta, \xi \in Y$, and so $\xi \mapsto f(\xi)-K \xi$ is globally Lipschitz with Lipschitz constant $\lambda$. Thus, it is clear that $F_{K}$ is globally Lipschitz with Lipschitz constant $1+\left\|\mathbf{G}^{K}\right\|_{H^{\infty}} \lambda$.

To show injectivity, assume that $F_{K}(\xi)=F_{K}(\zeta)$, where $\xi, \zeta \in Y$. Seeking a contradiction, suppose that $\xi \neq \zeta$. Then, by definition of $F_{K}$, we obtain

$$
\begin{aligned}
\|\xi-\zeta\|_{Y} & =\left\|\mathbf{G}^{K}(1)(f(\xi)-K \xi)-\mathbf{G}^{K}(1)(f(\zeta)-K \zeta)\right\|_{Y} \\
& \leq\left\|\mathbf{G}^{K}\right\|_{H^{\infty}}\|f(\xi)-f(\zeta)-K(\xi-\zeta)\|_{U} \\
& <\left\|\mathbf{G}^{K}\right\|_{H^{\infty}} \gamma\|\xi-\zeta\|_{Y} \\
& =\|\xi-\zeta\|_{Y}
\end{aligned}
$$

yielding a contradiction. It now follows that $\xi=\zeta$ and $F_{K}$ is injective.

We proceed to show surjectivity. To that end, observe that the map $Y \rightarrow Y, \xi \mapsto \mathbf{G}^{K}(1)(f(\xi)-$ $K \xi)$ is a contraction. Indeed, by (4.7),

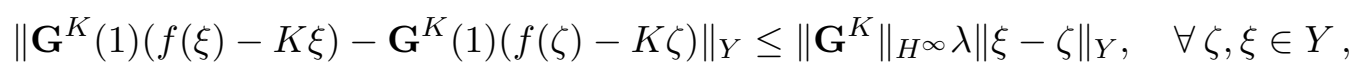

and, furthermore, $\left\|\mathbf{G}^{K}\right\|_{H^{\infty} \lambda}<\left\|\mathbf{G}^{K}\right\|_{H^{\infty}} \gamma=1$.

Fix $y \in Y$ and define the map $h_{y}: Y \rightarrow Y$ by

$$
h_{y}(z):=z-F_{K}(z)+y \quad \forall z \in Y .
$$

We note that $h_{y}$ is also a contraction since,

$$
\begin{aligned}
\left\|h_{y}(\xi)-h_{y}(\zeta)\right\|_{Y} & =\left\|\mathbf{G}^{K}(1)(f(\xi)-K \xi)-\mathbf{G}^{K}(1)(f(\zeta)-K \zeta)\right\|_{Y} \\
& \leq\left\|\mathbf{G}^{K}\right\|_{H^{\infty}} \lambda\|\xi-\zeta\|_{Y}, \quad \forall \xi, \zeta \in Y .
\end{aligned}
$$

Hence, by the contraction mapping theorem, there exists a (unique) fixed point of $h_{y}$, that is, there exists $x^{*} \in Y$ such that $h_{y}\left(x^{*}\right)=x^{*}$. This is equivalent to

$$
x^{*}-F_{K}\left(x^{*}\right)+y=x^{*},
$$

and so $F_{K}\left(x^{*}\right)=y$, showing that $F_{K}$ is surjective.

(iii) Let $\xi, \zeta \in Y$. Since $F_{K}$ is surjective, there exist $\eta_{1}, \eta_{2} \in Y$ such that

$$
F_{K}\left(\eta_{1}\right)=\xi, \quad F_{K}\left(\eta_{2}\right)=\zeta .
$$

Now, by definition of $F_{K}$ and using (4.7),

$$
\begin{aligned}
\left\|\eta_{1}-\eta_{2}\right\|_{Y} & =\left\|F_{K}\left(\eta_{1}\right)+\mathbf{G}^{K}(1)\left(f\left(\eta_{1}\right)-K \eta_{1}\right)-F_{K}\left(\eta_{2}\right)-\mathbf{G}^{K}(1)\left(f\left(\eta_{2}\right)-K \eta_{2}\right)\right\|_{Y} \\
& \leq\|\xi-\zeta\|_{Y}+\left\|\mathbf{G}^{K}\right\|_{H^{\infty}}\left\|f\left(\eta_{1}\right)-f\left(\eta_{2}\right)-K\left(\eta_{1}-\eta_{2}\right)\right\|_{Y} \\
& \leq\|\xi-\zeta\|_{Y}+\left\|\mathbf{G}^{K}\right\|_{H^{\infty}} \lambda\left\|\eta_{1}-\eta_{2}\right\|_{Y} .
\end{aligned}
$$


Now $F_{K}^{-1}(\xi)=\eta_{1}, F_{K}^{-1}(\zeta)=\eta_{2}, \lambda\left\|\mathbf{G}^{K}\right\|_{H^{\infty}}<1$ and we conclude that

$$
\left\|F_{K}^{-1}(\xi)-F_{K}^{-1}(\zeta)\right\|_{Y} \leq \frac{1}{1-\lambda\left\|\mathbf{G}^{K}\right\|_{H^{\infty}}}\|\xi-\zeta\|_{Y}
$$

completing the proof.

Proof of Lemma 4.2. Setting $f^{K}(\xi):=f(\xi)-K(\xi)$ and invoking definitions of $x^{\infty}$ and $y^{\infty}$, we obtain

$$
\begin{aligned}
C^{K} x^{\infty} & =C^{K}\left(I-A^{K}\right)^{-1}\left(B^{K}\left(f\left(z^{\infty}\right)-K\left(z^{\infty}-w^{\infty}\right)\right)+\left(B_{\mathrm{e}}+B^{K} K D_{\mathrm{e}}\right) v^{\infty}\right) \\
& =\mathbf{G}^{K}(1) f^{K}\left(z^{\infty}\right)-D^{K} f^{K}\left(z^{\infty}\right)+C^{K}\left(I-A^{K}\right)^{-1}\left(\left(B_{\mathrm{e}}+B^{K} K D_{\mathrm{e}}\right) v^{\infty}+B^{K} K w^{\infty}\right) \\
& =z^{\infty}-F_{K}\left(z^{\infty}\right)-D^{K} f^{K}\left(z^{\infty}\right)+C^{K}\left(I-A^{K}\right)^{-1}\left(\left(B_{\mathrm{e}}+B^{K} K D_{\mathrm{e}}\right) v^{\infty}+B^{K} K w^{\infty}\right) .
\end{aligned}
$$

Noting that $F_{K}\left(z^{\infty}\right)=C^{K}\left(I-A^{K}\right)^{-1}\left(B_{\mathrm{e}}+B^{K} K D_{\mathrm{e}}\right) v^{\infty}+(I-D K)^{-1} D_{\mathrm{e}} v^{\infty}+\left(I+\mathbf{G}^{K}(1) K\right) w^{\infty}$, it is easily seen that

$$
y^{\infty}=C^{K} x^{\infty}+D^{K}\left(f\left(y^{\infty}+w^{\infty}\right)-K y^{\infty}\right)+(I-D K)^{-1} D_{\mathrm{e}} v^{\infty},
$$

as required. To prove that $\left(v^{\infty}, w^{\infty}, x^{\infty}, y^{\infty}\right)$ is an equilibrium quadruple, we note that

$$
x^{\infty}=A^{K} x^{\infty}+B^{K}\left(f\left(y^{\infty}+w^{\infty}\right)-K y^{\infty}\right)+\left(B_{\mathrm{e}}+B^{K} K D_{\mathrm{e}}\right) v^{\infty} .
$$

Invoking Lemma 2.2 completes the proof.

\section{B Sampled-data example: further details}

We provide details on the numerical approximation used in Example 7.1. We first derive the weak form of (7.1), from which the finite-element approximation is computed. Given $z^{0} \in X$ and $v \in L_{\text {loc }}^{\infty}\left(\mathbb{R}_{+}\right)$, let $z$ denote a solution of (7.1). Multiplying both sides of the PDE in (7.1) by $\psi \in H^{1}(0,1)$, integrating over the spatial domain and integrating by parts gives

$$
\int_{0}^{1} z_{t}(\xi) \psi(\xi) d \xi=-\int_{0}^{1} z_{\xi}(\xi) \psi_{\xi}(\xi) d \xi+\left(2 \int_{1 / 2}^{1} \psi(\xi) d \xi\right) u+\left(\int_{0}^{1} b_{\mathrm{e}}(\xi) \psi(\xi) d \xi\right) v
$$

Observe that we do not need to impose any boundary conditions on the space of test functions, so we take $H^{1}(0,1)$ as the test function space. We seek an approximate solution to (B.1) of the form

$$
z_{N}(t, \xi)=\sum_{j=0}^{N} a_{j}(t) \phi_{j}(\xi), \quad \forall t>0, \forall \xi \in(0,1)
$$

where $N \in \mathbb{N}$ and $\phi_{j} \in H^{1}(0,1)$ are the usual (piecewise linear) hat or tent functions over the uniform mesh on $[0,1]$, and the $a_{i}(t)$ are scalar coefficients. Therefore, setting

$$
x:=\left(\begin{array}{llll}
a_{1} & a_{2} & \ldots & a_{N}
\end{array}\right)^{T},
$$

and taking $\psi=\phi_{i}$ for all $i \in\{0,1, \ldots, N\}$ in (B.1) yields

$$
M \dot{x}=-D x+F u+J v .
$$


Here $(M, D, F, J) \in \mathbb{R}^{(N+1) \times(N+1)} \times \mathbb{R}^{(N+1) \times(N+1)} \times \mathbb{R}^{(N+1)} \times \mathbb{R}^{(N+1)}$ have components

$$
\left.\begin{array}{rlrl}
M_{i j} & :=\int_{0}^{1} \phi_{i}(\xi) \phi_{j}(\xi) d \xi, & D_{i j}:=\int_{0}^{1} \phi_{i}^{\prime}(\xi) \phi_{j}^{\prime}(\xi) d \xi \\
F_{i}:=2 \int_{1 / 2}^{1} \phi_{i}(\xi) d \xi, & J_{i}:=\int_{0}^{1} b_{\mathrm{e}}(\xi) \phi_{i}(\xi) d \xi
\end{array}\right\} \forall i, j \in\{0,1, \ldots, N\} .
$$

The matrices $M, D$ and $F$ may be found analytically, whilst $J$ may have to be computed numerically. It is straightforward to show that $M=M^{T}>0$, and so we obtain the controlled linear system

$$
\dot{x}=-M^{-1} D x+M^{-1} F u+M^{-1} J w,
$$

with output

$$
y=L x, \quad \text { where } \quad L_{i}:=2 \int_{0}^{1 / 2} \phi_{i}(\xi) d \xi \quad \forall i \in\{0,1, \ldots, N\} .
$$

In our numerical simulations we take $N=30$. The resulting sampled-data ordinary differential equation is solved numerically using the Mathwork's MATLAB [32] command ode45, over each sampling period.

\section{IPM example: further details}

We provide details on the numerical approximation used in Example 7.2. For notational convenience in this section we set $\underline{N}:=\{1,2, \ldots, N\}$ for each $N \in \mathbb{N}$. To derive a finite element approximation of the forced IPM (7.7), we first derive a weak form. For which purpose, we multiply both sides of (7.7) by $\psi \in L^{1}(\Omega)$ and integrate over $\Omega$ to give

$$
\begin{aligned}
\int_{\Omega} \psi(\xi) x(t+1, \xi) d \xi= & \int_{\Omega} \psi(\xi)\left[(A x)(\xi)+b(\xi) f\left(c^{*} x\right)+b_{\mathrm{e}}(\xi) v(t)\right] d \xi \\
= & \int_{\Omega} \psi(\xi)\left(\int_{\Omega} p(\xi, \zeta) x(t, \zeta) d \zeta\right) d \xi+\left(\int_{\Omega} \psi(\xi) b(\xi) d \xi\right) f\left(c^{*} x\right) \\
& +\left(\int_{\Omega} \psi(\xi) b_{\mathrm{e}}(\xi) d \xi\right) v(t)
\end{aligned}
$$

We seek an approximate solution to (C.1) of the form

$$
x_{N}(t, \xi)=\sum_{j=1}^{N} a_{j}(t) \phi_{j}(\xi), \quad \forall t \in \mathbb{Z}_{+}, \quad \forall \xi \in \Omega,
$$

where $N \in \mathbb{N}, \phi_{j}$ are given $L^{1}$ functions and $a_{j}(t)$ are scalar coefficients. Substituting (C.2) into (C.1), and testing against $\psi=\phi_{i}$ for each $i \in \underline{N}$ gives

$$
\begin{aligned}
\sum_{j=0}^{N}\left(\int_{\Omega} \phi_{i}(\xi) \phi_{j}(\xi) d \xi\right) a_{j}(t+1)= & \sum_{j=0}^{N}\left(\int_{\Omega} \phi_{i}(\xi) \int_{\Omega} p(\xi, \zeta) \phi_{j}(\xi) d \xi\right) a_{j}(t) \\
& +\left(\int_{\Omega} \phi_{i}(\xi) b(\xi) d \xi\right) f\left(c^{*} x_{N}\right) \\
& +\left(\int_{\Omega} \phi_{i}(\xi) b_{\mathrm{e}}(\xi) d \xi\right) v(t) .
\end{aligned}
$$

Noting that

$$
c^{*} x_{N}(t, \cdot)=\sum_{j=0}^{N}\left(\int_{\Omega} c(\xi) \phi_{j}(\xi) d \xi\right) a_{j}(t), \quad \forall t \in \mathbb{Z}_{+},
$$


and setting

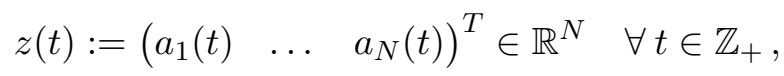

we see that (C.3) may be expressed in matrix form as

$$
z^{+}=M^{-1} D z+M^{-1} F f(L z)+M^{-1} J v .
$$

Here $\left(M, D, J, F, L^{T}\right) \in \mathbb{R}^{N \times N} \times \mathbb{R}^{N \times N} \times \mathbb{R}^{N} \times \mathbb{R}^{N} \times \mathbb{R}^{N}$ are given componentwise by

$$
\left.\begin{array}{rlrl}
M_{i j} & :=\int_{\Omega} \phi_{i}(\xi) \phi_{j}(\xi) d \xi, & F_{i} & :=\int_{\Omega} \phi_{i}(\xi) b(\xi) d \xi \\
J_{i} & :=\int_{\Omega} \phi_{i}(\xi) b_{\mathrm{e}}(\xi) d \xi, & L_{i} & :=\int_{\Omega} \phi_{i}(\xi) c(\xi) d \xi
\end{array}\right\} \quad \forall i, j \in \underline{N},
$$

and

$$
D_{i j}:=\int_{\Omega} \phi_{i}(\xi)\left(\int_{\Omega} p(\xi, \zeta) \phi_{j}(\zeta) d \zeta\right) d \xi, \quad \forall i, j \in \underline{N} .
$$

Since we are seeking to approximate the $L^{1}(\Omega)$ functions $x(t, \cdot)$ in $L^{1}(\Omega)$ (that is, we are not approximating any derivatives), we choose as finite-dimensional approximation spaces the linear span of $N$ piecewise constant functions. Specifically, for fixed $N \in \mathbb{N}$, we define $\xi_{j}:=m_{1}+$ $j\left(m_{2}-m_{1}\right) / N$ for $j \in\{0,1, \ldots, N\}, \Delta:=\left(m_{2}-m_{1}\right) / N$ and

$$
\phi_{i}: \Omega \rightarrow \mathbb{R}_{+}, \quad \phi_{i}(\xi):=\left\{\begin{array}{ll}
\frac{1}{\sqrt{\Delta}} & \xi_{i-1} \leq \xi \leq \xi_{i} \\
0 & \text { else, }
\end{array} \quad \forall i \in \underline{N} .\right.
$$

An advantage of such a choice is that, as readily seen, $M=I$, because $M_{i j}=0$ if $i \neq j$ and

$$
M_{i i}=\int_{m_{1}}^{m_{2}} \phi_{i}^{2}(\xi) d \xi=\frac{1}{\Delta} \int_{\xi_{i-1}}^{\xi_{i}} 1 d \xi=1, \quad \forall i \in \underline{N} .
$$

Consequently, (C.4) becomes

$$
z^{+}=D z+F f(L z)+J v .
$$

Moreover, by inspection of (C.5) and (C.6), it follows that with the above choice of piecewise constant $\phi_{j}$, the matrices $D, L, F$ and $J$ in (C.6) are componentwise nonnegative.

For the simulations in Example 7.2 we use (C.7) with $N=30$, and the matrices $D, L, J$ and $F$ are computed numerically.

\section{References}

[1] D. Angeli. A Lyapunov approach to incremental stability properties, IEEE Trans. Automatic Control, 47(3):410-421, 2002.

[2] M. Arcak \& A. Teel. Input-to-state stability for a class of Lurie systems, Automatica, 38(11):1945-1949, 2002.

[3] A. Bill, C. Guiver, H. Logemann \& S. Townley. Stability of nonnegative Lur'e systems. SIAM J. Control Optimiz., 54(3):1176-1211, 2016.

[4] A. Bill, C. Guiver, H. Logemann \& S. Townley. The converging-input convergingstate property for Lur'e systems. Math. Control Signals Systems, 29:4, 2017. https://doi.org/10.1007/s00498-016-0184-3

[5] J. Briggs, K. Dabbs, M. Holm, J. Lubben, R. Rebarber, B. Tenhumberg \& D. Riser-Espinoza. Structured population dynamics: an introduction to integral modeling. Mathematics Magazine, 83(4):243-257, 2010. 
[6] D. Z. Childs, M. Rees, K. E. Rose, P. J. Grubb \& S. P. Ellner. Evolution of complex flowering strategies: an age- and size-structured integral projection model. Proc. R. Soc. B-Bio. Sci., 270(1526):1829-1838, 2003.

[7] R. F. Curtain \& H. Zwart. An introduction to infinite-dimensional linear systems theory, Springer-Verlag, New York, 1995.

[8] S. N. Dashkovskiy, D. V. Efimov \& E. D. Sontag. Input-to-state stability and related properties of systems. Avtomat. i Telemekh., 72(8):3-40, 2011.

[9] S. N. Dashkovskiy \& A. Mironchenko. Input-to-state stability of infinite-dimensional control systems, Math. Control Signals Systems, 25(1):1-35, 2013.

[10] S. N. Dashkovskiy, B. S. Rüffer \& F. R. Wirth. An ISS small gain theorem for general networks. Math. Control Signals Systems, 19(2):93-122, 2007.

[11] E. A. Eager, R. Rebarber \& B. Tenhumberg. Choice of density-dependent seedling recruitment function affects predicted transient dynamics: a case study with Platte thistle. Theor. Ecol., 5(3):387-401, 2012.

[12] M. R. Easterling, S. P. Ellner \& P. M. Dixon. Size-specific sensitivity: applying a new structured population model. Ecology, 81(3):694-708, 2000.

[13] S. P. Ellner \& M. Rees. Integral projection models for species with complex demography. Am. Nat., 167(3):410-428, 2006.

[14] D. Franco, C. Guiver, H. Logemann \& J. Peràn. A class of forced nonlinear infinitedimensional population models: boundedness, persistence and stability. Submitted, 2018.

[15] C. Guiver, H. Logemann \& M. R. Opmeer. Transfer functions of infinite-dimensional systems: positive realness and stabilization. Math. Control Signals Systems., 29:20, 2017. https://doi.org/10.1007/s00498-017-0203-z

[16] C. Guiver, H. Logemann \& M. R. Opmeer. Infinite-dimensional Lur'e systems: input-tostate stability and convergence properties. SIAM J. Control Optim., 57(1):334-365, 2019.

[17] D. Hinrichsen \& A. J. Pritchard. Mathematical systems theory I, Springer-Verlag, Berlin, 2005.

[18] B. Jacob, R. Nabiullin, J. R. Partington \& F. L. Schwenninger. Infinite-dimensional inputto-state stability and Orlicz spaces. SIAM J. Control Optim., 56(2):868-889, 2018.

[19] B. Jayawardhana, H. Logemann \& E. P. Ryan. Input-to-state stability of differential inclusions with applications to hysteretic and quantized feedback systems. SIAM J. Control Optim., 48(2):1031-1054, 2009.

[20] B. Jayawardhana, H. Logemann \& E. P. Ryan. The circle criterion and input-to-state stability: new perspectives on a classical result. IEEE Control Syst. Mag., 31(4):32-67, 2011.

[21] Z.-P. Jiang, A. R. Teel \& L. Praly. Small-gain theorem for ISS systems and applications. Math. Control Signals Systems, 7(2):95-120, 1994.

[22] Z.-P. Jiang \& Y. Wang. Input-to-state stability for discrete-time nonlinear systems. Automatica, 37(6):857-869, 2001.

[23] Z.-P. Jiang \& Y. Wang. A converse Lyapunov theorem for discrete-time systems with disturbances. Systems Control Lett., 45(1):49-58, 2002.

[24] Z.-P. Jiang \& Y. Wang. Nonlinear small-gain theorems for discrete-time feedback systems and applications. Automatica, 40(12):2125-2136, 2005.

[25] H. K. Khalil. Nonlinear systems. 3rd ed., Prentice-Hall, Upper Saddle River, 2002.

[26] P. D. Lax. Functional analysis. John Wiley \& Sons, New York, 2002.

[27] M. R. Liberzon. Essays on the absolute stability theory. Automation and remote control, 67(10):1610-1644, 2006. 
[28] H. Logemann. Stabilization of well-posed infinite-dimensional systems by dynamic sampleddata feedback. SIAM J. Control Optim., 51(2):1203-1231, 2013.

[29] H. Logemann, R. Rebarber \& S. Townley. Stability of infinite-dimensional sampled-data systems. Trans. Amer. Math. Soc., 355(8):3301-3328, 2003.

[30] H. Logemann, R. Rebarber \& S. Townley. Generalized sampled-data stabilization of wellposed linear infinite-dimensional systems. SIAM J. Control Optim., 44(4):1345-1369, 2005.

[31] H. Logemann, E. P. Ryan \& S. Townley. Integral control of linear systems with actuator nonlinearities: lower bounds for the maximal regulating gain. IEEE Trans. Automat. Control, 44(6):1315-1319, 1999.

[32] MATLAB Release 2014b, The MathWorks, Inc., Natick, Massachusetts, United States.

[33] C. Merow, J.P. Dahlgren, C.J.E. Metcalf, D.Z. Childs, M.E. Evans, E. Jongejans, S. Record, M. Rees, R. Salguero-Gómez \& S.M. McMahon. Advancing population ecology with integral projection models: a practical guide. Methods Ecol. Evol., 5(2):99-110, 2014.

[34] A. Mironchenko, I. Karafyllis \& M. Krstic. Monotonicity methods for input-to-state stability of nonlinear parabolic PDEs with boundary disturbancess, (2017), arXiv:1706.07224.

[35] A. Mironchenko \& F. Wirth. Characterizations of input-to-state stability for infinitedimensional systems, IEEE Trans. Automat. Control, 63(6):1692-1707, 2018.

[36] A. Pazy. Semigroups of linear operators and applications to partial differential equations. Springer-Verlag, New York, 1983.

[37] P. Pepe \& Z. P. Jiang. A Lyapunov-Krasovskii methodology for ISS and iISS of time-delay systems. Systems Control Lett., 55(12):1006-1014, 2006.

[38] C. Prieur \& F. Mazenc. ISS-Lyapunov functions for time-varying hyperbolic systems of balance laws. Math. Control Signals Systems, 24(1-2):111-134, 2012.

[39] K. E. Rose, S. M. Louda \& M. Rees. Demographic and evolutionary impacts of native and invasive insect herbivores on Cirsium canescens. Ecology, 86(2):453-465, 2005.

[40] I. G. Rosen \& C. Wang. On stabilizability and sampling for infinite-dimensional systems. IEEE Trans. Automat. Control, 37(10):1653-1656, 1992.

[41] W. Rudin. Real and complex analysis, 3rd edition. McGraw-Hill, New York, 1987.

[42] E. Sarkans \& H. Logemann. Input-to-state stability of Lur'e systems Math. Control Signals Systems, 27(4):439-465, 2015.

[43] E. Sarkans \& H. Logemann. Stability of higher-order discrete-time Lur'e systems Linear Algebra Appl., 506:183-211, 2016.

[44] E. Sarkans \& H. Logemann. Input-to-state stability of discrete-time Lur'e systems SIAM J. Control Optim., 54(3):1739-1768, 2016.

[45] E. D. Sontag. Smooth stabilization implies coprime factorization. IEEE Trans. Automat. Control, 34(4):435-443, 1989.

[46] E. D. Sontag. Comments on integral variants of ISS, Systems Control Lett., 34(1-2):93-100, 1998.

[47] E. D. Sontag. Input to state stability: basic concepts and results. In Nonlinear and optimal control theory, volume 1932 of Lecture Notes in Math., pages 163-220. Springer, Berlin 2008.

[48] E. D. Sontag \& Y. Wang. On characterizations of the input-to-state stability property, Systems Control Lett., 24(5):351-359, 1995.

[49] E. D. Sontag \& Y. Wang. New characterizations of input-to-state stability. IEEE Trans. Automat. Control, 41(9):1283-1294, 1997.

[50] M. Vidyasagar. Nonlinear systems analysis SIAM, Philadelphia, PA, 2002. 